\title{
Research Article Adaptive Output Tracking of Driven Oscillators
}

\author{
Lili Diao and Martin Guay \\ Chemical Engineering Department, Queen's University, Kingston, ON, Canada K7L 3N6 \\ Correspondence should be addressed to Martin Guay, guaym@chee.queensu.ca
}

Received 1 August 2007; Revised 13 May 2008; Accepted 1 August 2008

Recommended by Jing Sun

Heart dynamics are usually unknown and require the application of real-time control technique because of the fatal nature of most cardiac arrhythmias. The problem of controlling the heart dynamics in a real-time manner is formulated as an adaptive learning output-tracking problem. For a class of nonlinear dynamic systems with unknown nonlinearities and nonaffine control input, a Lyapunov-based technique is used to develop a control law. An adaptive learning algorithm is exploited that guarantees the stability of the closed-loop system and convergence of the output tracking error to an adjustable neighborhood of the origin. In addition, good approximation of the unknown nonlinearities is also achieved by incorporating a persistent exciting signal in the parameter update law. The effectiveness of the proposed method is demonstrated by an application to a cardiac conduction system modelled by two coupled driven oscillators.

Copyright (C) 2008 L. Diao and M. Guay. This is an open access article distributed under the Creative Commons Attribution License, which permits unrestricted use, distribution, and reproduction in any medium, provided the original work is properly cited.

\section{INTRODUCTION}

Heart dynamics are very complicated by nature, and it is widely known that accurate analytical models are difficult to develop for cardiac dynamics and different types of arrhythmias. In addition, real-time control technique is needed because of the fatal nature of cardiac arrhythmias. As a result, real-time model-independent control techniques are needed to control heart dynamics in the presence of cardiac arrhythmias.

The dynamics of cardiac arrhythmias have been closely related to a variety of bifurcations and chaos phenomena. In the recent years [1-4], the theory of chaos control has made contributions to a mechanistic understanding of cardiac arrhythmias. Without detailed knowledge of the heart dynamics model structure, chaos control technique is able to regulate the abnormal heart rhythm by stabilizing the system around a desirable limit cycle. However, this approach is limited because to find a suitable controller parameter, it has to go through a "learning stage," which comprises precontrol time-series recording and system dynamics estimation. In addition, the "learning stage" based on previous time series can lead to a poor estimation of system dynamics, because of the evolving nature of biological systems. The available adaptive approach [5] concentrated on linear chaos control, which is limited because the linear approach, can only delay or change the bifurcation location, while nonlinear control is needed in order to modify the stability property. According to the above observation, chaos control cannot serve as a real-time control technique to regulate cardiac arrhythmias.

To overcome this difficulty, we propose to apply some adaptive control technique to control cardiac arrhythmias. Rather than treat the unknown heart dynamics as a "black box," we try to estimate the unknown dynamics using a neural network (NN) approach. NN techniques have undergone great developments and have been successfully applied in many fields, such as pattern recognition, signal processing, modelling, and system control. The approximating ability of $\mathrm{NN}$ has been proven in [6]. Multilayer NN identification and control techniques have been developed and demonstrated through simulation $[7,8]$, following the popularization of the "backpropagation" algorithm. However, analytical results obtained in [9] show that offline training is needed, because stability can be guaranteed only when the initial network weights are chosen sufficiently close to the ideal weight. To avoid the above difficulties in constructing stable neural systems, Lyapunov stability theory has been applied in developing control structure and deriving network weight updating laws [10-12]. Recently, multilayer $\mathrm{NN}$ control has been successfully applied to robotic control $[13,14]$. In addition, [15] provides a systematic treatment of common problems in robotic control by introducing the G-Lee operator. 
The aforementioned NN approaches are restricted to control-affine nonlinear systems. The problem of adaptively controlling systems with unknown nonaffine input nonlinearities is still open in the literature. Heart dynamics generally fall into the category of nonaffine systems, because of the inadequate knowledge of heart dynamics, and limited understanding of how actuators enter the dynamics.

Another limitation of current approaches is that approximation performance of the unknown nonlinearity and parameter estimation convergence are not discussed. Usually a high-gain control is employed to dominate the approximation error to ensure good tracking performance. However, in reality, it is often desirable to identify the unknown part of the dynamics.

In this work, we focus on an adaptive learning technique that is applicable to unknown nonlinear dynamic plants with a class of nonaffine input uncertainties, that are unknown, but continuous, and satisfy a sector constraint. An external signal, which is designed to be persistent exciting, is imbedded in the parameter update law to ensure good approximation and parameter convergence.

This paper is organized as follows. Section 2 presents the proposed adaptive controller. Application of the adaptive output feedback tracking technique to cardiac dynamics control is provided in Section 3. In Section 4, brief conclusions of the paper are given.

\section{ADAPTIVE CONTROL DESIGN}

We consider single-input/single-output (SISO) controllable nonlinear systems of the form

$$
\begin{aligned}
\dot{\xi} & =\phi(\xi, z), \\
\dot{z}_{1} & =z_{2}, \\
\vdots & \\
\dot{z}_{\rho} & =f(z, \xi, u), \\
y & =z_{1},
\end{aligned}
$$

where $\xi=\left[\xi_{1}, \xi_{2}, \ldots, \xi_{n-\rho}\right]^{T} \in \mathbb{R}^{n-\rho}$ are the state variables of the zero dynamics; $z=\left[z_{1}, z_{2}, \ldots, z_{\rho}\right]^{T} \in \mathbb{R}^{\rho}$ are the state variables of the main dynamics; $u \in \mathbb{R}$ and $y \in \mathbb{R}$ are the system input and output, respectively. The mapping $f(z, \xi, u)$ is assumed to be an unknown continuous function of $z$ and $u$ and is assumed to be globally Lipschitz in $\xi$. Given a reference trajectory $y_{r}$, the control objective is to design an output feedback controller for system (1), which achieves good tracking performance subject to the unknown nonlinearities in the system.

Let $Y_{r}=\left[y_{r}, \dot{y}_{r}, \ldots, y_{r}^{(\rho-1)}\right]^{T}$. In this work, it is assumed that $\left\|Y_{r}\right\| \leq c,\left|y_{r}^{(\rho)}\right| \leq \bar{\nu}_{1}$, with known constant $c>$ $0, \bar{\nu}_{1}>0$. Denote $\xi_{r}$ the "steady-state" response of the tracking zero dynamics, governed by the differential equation $\dot{\xi}_{r}=\phi\left(\xi_{r}, Y_{r}\right)$, and $\bar{\xi}=\xi-\xi_{r}$. It is also assumed that $\left\|\xi_{r}\right\| \leq c_{\xi}$, where $c_{\xi}>0$ is a known constant. Denote
$e=z-Y_{r}=\left[e_{1}, e_{2}, \ldots, e_{\rho}\right]^{T}, e_{c}=\left[e_{1}, e_{2}, \ldots, e_{\rho-1}\right]^{T}$, and $e_{s}=\Lambda^{T} e_{c}+e_{\rho}$, where $\Lambda=\left[\lambda_{1}, \lambda_{2}, \ldots, \lambda_{\rho-1}\right]$ is to be chosen.

In this paper, radial basis function (RBF) presented in [16] was used to approximate a continuous function $\psi(x)$ : $\mathbb{R}^{p} \rightarrow \mathbb{R}$

$$
\psi(x)=W^{*} S(x)+\mu_{l}(x),
$$

with approximation error $\mu_{l}(x)$, and basis function vector

$$
\begin{aligned}
& S(x)=\left[s_{1}(x), s_{2}(x), \ldots, s_{l}(x)\right]^{T}, \\
& s_{i}(x)=\exp \left[\frac{-\left(x-\varphi_{i}\right)^{T}\left(x-\varphi_{i}\right)}{\sigma_{i}^{2}}\right], \quad i=1,2, \ldots, l,
\end{aligned}
$$

where $\varphi_{i}$ is the center of the receptive field, and $\sigma_{i}$ is the width of the Gaussian function. The ideal weight $W^{*}$ in (2) is defined as

$$
W^{*}:=\arg \min _{W \in \Omega_{w}}\left\{\sup _{x \in \Omega}\left|W^{T} S(x)-\psi(x)\right|\right\},
$$

where $\Omega_{w}=\left\{W \mid\|W\| \leq w_{m}\right\}$ with positive constant $w_{m}$ to be chosen at the design stage, and $\Omega$ is a compact set. Universal approximation results stated in $[16,17]$ (and the references therein) indicate that if $l$ is chosen sufficiently large, then $W^{* T} S(x)$ can approximate any continuous function to any desired accuracy on a compact set, given that the centers are chosen close enough.

A number of assumptions are made for system (1).

Assumption 1. The sign of $\partial f\left(z, \xi_{r}, u\right) / \partial u$ is known, and there exist a positive constant $\bar{b}_{0}$ and a nonzero continuous function $\bar{b}_{1}(z)$ such that

$$
0<\bar{b}_{0} \leq\left|\frac{\partial f\left(z, \xi_{r}, u\right)}{\partial u}\right| \leq \bar{b}_{1}(z) .
$$

Assumption 2. The approximation error satisfies $\left|\mu_{l}(x(t))\right| \leq$ $\bar{\mu}_{l}$ with unknown constant $\bar{\mu}_{l}>0$ over a compact set.

The design task is achieved in two steps: firstly, a state feedback adaptive tracking controller is designed; secondly, a high gain observer is used together with the state feedback controller to yield an output feedback adaptive tracking control law. We propose the following adaptive controller design.

Given the reference trajectory $Y_{r}$ and $\xi_{r}$, system (1) can be rewritten in terms of the tracking error $e$ and $\bar{\xi}=\xi-\xi_{r}$ as follows:

$$
\begin{aligned}
\dot{\bar{\xi}} & =\phi\left(\bar{\xi}+\xi_{r}, e+Y_{r}\right)-\phi\left(\xi_{r}, Y_{r}\right), \\
\dot{e}_{c} & =A_{c} e_{c}+B_{c} e_{\rho}, \\
\dot{e}_{\rho} & =f\left(e+Y_{r}, \bar{\xi}+\xi_{r}, u\right)-y_{r}^{(\rho)},
\end{aligned}
$$

where

$$
A_{c}=\left[\begin{array}{ccccc}
0 & 1 & \ldots & \ldots & 0 \\
0 & 0 & 1 & \ldots & 0 \\
\vdots & \vdots & \vdots & \ddots & \vdots \\
0 & 0 & \ldots & \ldots & 1 \\
0 & 0 & \ldots & \ldots & 0
\end{array}\right], \quad B_{c}=\left[\begin{array}{c}
0 \\
0 \\
\vdots \\
0 \\
1
\end{array}\right]
$$


The proof of stability (convergence of the tracking error) is achieved by considering the tracking dynamics (6) and the error dynamics (7) as an interconnected system. With proper constraint imposed on the interconnected term, the overall stability is guaranteed by the use of small gain theorem [18], together with a proper choice of control and parameter update law. First, we must make the following assumption concerning system (6).

Assumption 3. The tracking dynamics of the system given by (6) are input-to-state stable (ISS). That is, there exists a positive definite function $U(\bar{\xi})$, such that the following is satisfied:

$$
\begin{gathered}
c_{1}\|\bar{\xi}\|^{2} \leq U(\bar{\xi}) \leq c_{2}\|\bar{\xi}\|^{2}, \\
\dot{U}(\bar{\xi}) \leq-c_{3}\|\bar{\xi}\|^{2}+c_{4}\|\bar{\xi}\|\|e\|,
\end{gathered}
$$

where $c_{1}, c_{2}, c_{3}$, and $c_{4}$ are positive constants, and $\dot{U}(\bar{\xi})$ is the time derivative of $U(\bar{\xi})$ along the solution of (1).

Considering $e$ as a "disturbance" to the tracking error zero dynamics (6), Assumption 3 ensures that $\bar{\xi}$ dynamics in (6) are input-to-state stable (ISS) with respect to $e$.

By $\partial f\left(z, \xi_{r}, u\right) / \partial u \neq 0, \forall z \in \mathbb{R}^{\rho}, \forall u \in \mathbb{R}$ (Assumption $2)$, it follows from the implicit function theorem [19] that there exists a continuous function $\alpha\left(z, \xi_{r}\right)$ such that $f\left(z, \xi_{r}, \alpha\left(z, \xi_{r}\right)\right)=0$. The function $f(z, \xi, u)$ may be reexpressed as

$$
\begin{aligned}
f(z, \xi, u)= & f\left(z, \xi_{r}, u\right)+f(z, \xi, u)-f\left(z, \xi_{r}, u\right) \\
= & f\left(z, \xi_{r}, \alpha\left(z, \xi_{r}\right)\right)+\int_{0}^{1} \frac{\partial f\left(z, \xi_{r}, u_{\lambda}\right)}{\partial u_{\lambda}} d \lambda\left(u-\alpha\left(z, \xi_{r}\right)\right) \\
& +\left[f(z, \xi, u)-f\left(z, \xi_{r}, u\right)\right] \\
= & b\left(z, \xi_{r}, u\right)\left(u-\alpha\left(z, \xi_{r}\right)\right)+\left[f(z, \xi, u)-f\left(z, \xi_{r}, u\right)\right]
\end{aligned}
$$

where $u_{\lambda}=\lambda u+(1-\lambda) \alpha\left(z, \xi_{r}\right), b\left(z, \xi_{r}, u\right)=\int_{0}^{1}\left(\partial f\left(z, \xi_{r}, u_{\lambda}\right) /\right.$ $\left.\partial u_{\lambda}\right) d \lambda$, and the following is assumed:

$$
\left|f(z, \xi, u)-f\left(z, \xi_{r}, u\right)\right| \leq L_{1}\|\bar{\xi}\|,
$$

where $L_{1}$ is a Lipschitz constant.

Approximate the unknown function $\alpha(z)$ as

$$
\alpha\left(z, \xi_{r}\right)=W^{* T} S\left(z, \xi_{r}\right)+\mu_{1}(x(t)) .
$$

Let $\widehat{W}$ denote the estimate of $W^{*}$. The parameter estimation error is given by $\widetilde{W}=\widehat{W}-W^{*}$.

Note that the boundedness of $\left|\partial f\left(z, \xi_{r}, u\right) / \partial u\right|$ (Assumption 2) implies that $b\left(z, \xi_{r}, u\right)$ is bounded as follows:

$$
b_{0} \leq b\left(z, \xi_{r}, u\right) \leq b_{1}\left(z, \xi_{r}\right) \text {, }
$$

where $b_{0}$ is a positive constant, and $b_{1}\left(z, \xi_{r}\right)$ is a nonzero continuous function.
Using (12), the error dynamics (7) can be written as

$$
\begin{aligned}
& \dot{e}_{c}=A_{c} e_{c}+B_{c} e_{\rho}, \\
& \dot{e}_{\rho}=b\left(z, \xi_{r}, u\right)(u-\alpha(z))-y_{r}^{(\rho)}+\left[f(z, \xi, u)-f\left(z, \xi_{r}, u\right)\right] .
\end{aligned}
$$

The state feedback design and parameter update law are given by

$$
\begin{gathered}
u=k\left(z, \xi_{r}\right) e_{s}+\widehat{W}^{T} S\left(z, \xi_{r}\right), \\
\dot{\widehat{W}}=\gamma_{w} \operatorname{Proj}\left(\widehat{W}, c(t) e_{s}\right),
\end{gathered}
$$

where $k\left(z, \xi_{r}\right)$ is the controller gain function, $\gamma_{w}$ is a positive constant, and $\operatorname{Proj}(\cdot)$ is a projection algorithm.

The dynamics of $c(t)$ are chosen as follows:

$$
\begin{aligned}
\dot{c}^{T}(t) & =-\left(b_{1} k_{t}-\frac{1}{2} B_{c}^{T} P B_{c}\right) c^{T}(t)-b_{1} S^{T}\left(z, \xi_{r}\right) \\
& =-K(t) c^{T}(t)+B(t),
\end{aligned}
$$

where $K(t)=b_{1} k_{t}-(1 / 2) B_{c}^{T} P B_{c}>0$, and $B(t)=$ $-b_{1} S^{T}\left(z, \xi_{r}\right)$. Note that $K(t)$ can always be made negative by a suitable choice of the gain constant $k_{t}$. The matrix $P$ is a positive definite solution of the Riccati-like equation:

$$
P A_{c}+A_{c}^{T} P+2 \gamma_{1} P B_{c} B_{c}^{T} P+\frac{1}{2} k_{2} A_{c}^{T} A_{c}+Q=0,
$$

for some positive definite symmetric matrix $Q$ and positive $\gamma_{1}>0$ and $k_{2}>0$ chosen as part of the design.

In addition to the above assumptions, we must ensure that a certain persistence of excitation condition is met to ensure that the unknown nonlinearity is estimated correctly.

Assumption 4. There exist positive constant $T>0$ and $k_{N}>0$ such that

$$
\int_{t}^{t+T} c(\tau) c^{T}(\tau) d \tau \geq k_{N} I_{N}
$$

where $c^{T}(t)$ is the solution of $(17), I_{N}$ is a $N$-dimensional identity matrix.

The following lemma will be used in the sequel.

Lemma 1. Consider the differential equation

$$
\dot{z}(t)=-\phi(t) \phi^{T}(t) z(t),
$$

where $z(t) \in \mathbb{R}_{+} \rightarrow \mathbb{R}^{n}$ and $\phi(t) \in \mathbb{R}_{+} \rightarrow \mathbb{R}^{n}$ are both column vectors. Assume that there exist $a T>0$ and $a k>0$ such that

$$
\int_{t}^{t+T} \phi(\tau) \phi^{T}(\tau) d \tau \geq k I
$$

then the origin of (20) is a globally exponentially stable equilibrium of the system.

The proof of this lemma can be found in [20].

Theorem 1 gives the main results for the state feedback controller design. 
Theorem 1. Consider the nonlinear system (1) in closed loop with the controller and parameter update law provided in (15) and (16). Assume that the signal $c(t)$ is such that

$$
\int_{t}^{t+T} c(\tau) c^{T}(\tau) d \tau \geq k_{N} I_{N}
$$

for positive constants $T>0$ and $k_{N}>0$, where $c^{T}(t)$ is a solution of (17).

Given Assumptions 1 to 3, all the signals of the closedloop system are bounded. The parameter estimation errors $\widetilde{W}$ converge exponentially to a small neighborhood of the origin.

The mean square tracking error satisfies

$$
\frac{1}{t} \int_{0}^{t} e_{1}^{2} d t \leq \frac{\alpha_{0}}{t} V_{s}(0)+\frac{1}{k}\left(\bar{\mu}_{l}^{2}+\bar{\nu}_{1}^{2}+w_{m}^{2}\right),
$$

where $\alpha_{0}$ is a positive constant, and $V_{s}(0)$ is a positive constant depending on system initial conditions.

Furthermore, the tracking error is such that

$$
\|e(t)\| \leq \alpha_{1} e^{-\beta_{1}\left(t-t_{0}\right)}+\frac{\alpha_{2}}{\sqrt{k}}\left(\bar{\mu}_{l}^{2}+\bar{\nu}_{1}^{2}+w_{m}^{2}\right) .
$$

where $\alpha_{1}, \alpha_{2}, \beta_{1}$, and $k$ are some positive constants.

Proof. See the appendix.

Since in practice, only a limited number of measurements can be obtained, one needs to build an observer to estimate the unmeasured states and implement the state feedback controller with the estimated states. For the tracking error system (7), a high-gain observer [19] is used, which takes the following form:

$$
\begin{gathered}
\dot{\hat{e}}_{1}=\hat{e}_{2}+\frac{l_{1}}{\epsilon}\left(e_{1}-\hat{e}_{1}\right), \\
\dot{\hat{e}}_{2}=\widehat{e}_{3}+\frac{l_{2}}{\epsilon^{2}}\left(e_{1}-\hat{e}_{1}\right), \\
\vdots \\
\hat{\hat{e}}_{\rho}=\frac{l_{\rho}}{\epsilon^{\rho}}\left(e_{1}-\hat{e}_{1}\right),
\end{gathered}
$$

where $\left[l_{1}, l_{2}, \ldots, l_{\rho}\right]^{T}$ are the coefficients of a Hurwitz polynomial, $\epsilon$ is some small positive constant, and $\hat{e}_{i}, i=1, \ldots, \rho$ are the estimated tracking errors.

Following [21], we define the scaled estimation errors:

$$
\eta_{i}=\frac{e_{i}-\hat{e}_{i}}{\epsilon^{n-i}}=\frac{\tilde{e}_{i}}{\epsilon^{n-i}}, \quad 1 \leq i \leq n .
$$

Using (6) and (25), the dynamics of the scaled estimation errors are given by

$$
\epsilon \dot{\eta}=A_{0} \eta+\epsilon B_{0}\left(y_{d}^{n}-f(x, u)\right),
$$

where the matrix $A_{0} \in \mathbb{R}^{n \times n}$ and the vector $B_{0} \in \mathbb{R}^{n}$ assume the following form:

$$
A_{0}=\left[\begin{array}{ccccc}
-\alpha_{1} & 1 & \ldots & \ldots & 0 \\
-\alpha_{2} & 0 & 1 & \ldots & 0 \\
\vdots & \vdots & \vdots & \ddots & \vdots \\
-\alpha_{n-1} & 0 & \ldots & \ldots & 1 \\
-\alpha_{n} & 0 & \ldots & \ldots & 0
\end{array}\right], \quad B_{0}=\left[\begin{array}{c}
0 \\
0 \\
\vdots \\
0 \\
1
\end{array}\right]
$$

We define the Lyapunov function $W(\eta)=(1 / 2) \eta^{T} P_{0} \eta$, where $P_{0}$ is the symmetric positive definite matrix solution of

$$
P_{0} A_{0}+A_{0}^{T} P_{0}=-I
$$

Let $V_{2}=(1 / 2) e_{c}^{T} P e_{c}+(1 / 2) e_{s}^{2}+\left(1 / 2 \gamma_{w}\right) \widetilde{W}^{T} \widetilde{W}$ and consider the compact sets $\Omega=\left\{e \in \mathbb{R}^{n}, \widetilde{W} \in \mathbb{R}^{l} \mid V_{2} \leq c_{1}\right\}$, where $c_{1}>\left(\left(1 / 2 k_{d}\right) \bar{v}_{1}^{2}+\left(1 / 2 k_{\mu}\right) \bar{\mu}_{l}^{2}+\left(2 / k_{w}+2 / \gamma\right) w_{m}^{2}\right)=c_{2}$. Define the positive constant $b$ such that $0<c_{2} \leq b<c_{1}$ and the set $\Omega_{b}=\left\{V_{2}(e, \widetilde{W}) \leq b\right\}$. For the estimation errors, we define the set $\Sigma=\left\{W(\eta) \leq \rho \epsilon^{2}\right\}$.

In order to apply the result of [21], we must verify that the state-feedback equation (15) and the learning rate for parameter estimation defined in (16) are globally bounded. Since they are not, we consider the application of a state feedback over the compact set $\Omega$. We first compute the constants

$$
\begin{gathered}
S_{u}>\max _{e, \widetilde{W} \in \Omega}\left(-k(x) e_{s}+\widehat{W}^{T} S(x)\right), \\
S_{\delta}>\max _{e, \widetilde{W} \in \Omega}\left(b_{1}(x) S(x) e_{s}\right) .
\end{gathered}
$$

The maximization is performed over all $(e, \widetilde{W}) \in \Omega, Y_{d} \in$ $Y$. It is assumed that the reference trajectories $Y_{d}=$ $\left[y_{d}, \dot{y}_{d}, \ldots, y_{d}^{(n-1)}\right]$ are bounded and evolve in a compact subset $Y$ of $\mathbb{R}^{n}$. This ensures that the state $x$ of the system are also bounded on $\Omega \times Y$. The state-feedback and the adaptive learning rate can then bounded on $\Omega$ by implementing the functions

$$
\begin{gathered}
\Psi_{s}\left(e, Y_{d}, \widetilde{W}\right)=S_{u} \operatorname{sat}\left(\frac{-k(x) e_{s}+\widehat{W}^{T} S(x)}{S_{u}}\right), \\
\Upsilon_{s}\left(e, Y_{d}, \widetilde{W}\right)=S_{\delta} \operatorname{sat}\left(\frac{b_{1}(x) S(x) e_{s}}{S_{\delta}}\right),
\end{gathered}
$$

where

$$
\text { sat }(w)= \begin{cases}-1, & \text { if } w \leq-1, \\ w, & \text { if }-1<w<1 \\ 1, & \text { if } w \geq 1\end{cases}
$$


The adaptive learning state feedback is rewritten as

$$
\begin{aligned}
& \dot{\widehat{W}}=\left\{\begin{array}{rlrl}
-\gamma \Upsilon_{s}, & \text { if }\|\widehat{W}\| & <w_{m}, & \text { or } \\
\|\widehat{W}\| & =w_{m}, \widehat{W}^{T} \Upsilon_{s} \geq 0, \\
-\gamma \Upsilon_{s}+\gamma \frac{\widehat{W} \widehat{W}^{T} \Upsilon_{s}}{\|\widehat{W}\|^{2}}, & \text { if }\|\widehat{W}\| & =w_{m}, & \widehat{W}^{T} \Upsilon_{s}<0,
\end{array}\right. \\
& u=\Psi_{s}\left(e, Y_{d}, \widetilde{W}\right)
\end{aligned}
$$

for $e, \widetilde{W} \in \Omega$. Having bounded the control and the adaptive learning rate, we pose the output-feedback controller

$$
\begin{aligned}
& \dot{\hat{e}}_{i}=\hat{e}_{i+1}+\frac{\alpha_{i}}{\epsilon^{i}} \widetilde{e}_{1}, \quad 1 \leq i \leq n-1, \\
& \dot{\hat{e}}_{n}=\frac{\alpha_{n}}{\epsilon^{n}} \widetilde{e}_{1}, \\
& \dot{\widehat{W}}=\left\{\begin{array}{lr}
-\gamma \hat{\Upsilon}_{s}, & \text { if }\|\widehat{W}\|<w_{m}, \text { or } \\
-\gamma \hat{\Upsilon}_{s}+\gamma \frac{\widehat{W} \widehat{W}^{T} \hat{\Upsilon}_{s}}{\|\widehat{W}\|^{2}}, & \text { if }\|\widehat{W}\|=w_{m}, \widehat{W}^{T} \hat{\Upsilon}_{s}<0 \\
u & =\Psi_{s}\left(\hat{e}, Y_{d}, \widehat{W}\right),
\end{array}\right.
\end{aligned}
$$

where $\hat{\Upsilon}_{s}=\Upsilon_{s}\left(\hat{e}, Y_{d}\right)$.

Theorem 2 provides the result for output feedback controller design.

Theorem 2. Consider the nonlinear system equation (1). If Assumptions 1 to 3 are met then the dynamic output feedback controller equations (35)-(37) guarantee that for any initial conditions of the closed-loop system starting in $S \times O$ there exists $0<\epsilon<\epsilon_{3}^{*}$ such that every trajectory of the closed-loop system enters a small neighborhood of the origin in finite time, and it converges exponentially to a small adjustable neighborhood of the origin.

Proof. See the appendix.

The proof of Theorem 2 is included in the appendices for the sake of completeness. The approach is very similar to the one in the state-feedback case, except that a saturation function is used to isolate the peaking phenomenon in the estimated state dynamics, so as not to cause instability in the original state dynamics.

Remark 1. In many applications, convergence of the error dynamics to a small neighbourhood of the origin may prove to be a significant limitation. One mechanism that is known to reduce the onset of tracking error offsets is the addition of integral action. In the current context, it is straightforward to include the integral term, $\dot{e}_{0}=e_{1}$, which guarantees convergence of the tracking error.

\section{APPLICATION TO A DRIVEN OSCILLATOR SYSTEM}

In the literature discussing the problem of regulating cardiac arrhythmias via chaos control, different types of cardiac models have been used. Some are merely constructed to describe a particular type of arrhythmias, such as the irregular interbeat model [22]. The others are models for different functions in the heart, such as the "black box" models [4] and empirical model [23] for the AV conduction system, and the mechanistic model that accounts for the action potentials of the ventricular myocardium [22].

One distinct physical mechanism in the heart is the pacemaker, consisting of the sinoatrial (SA) node and the atrioventricular (AV) node. The idea of considering this system mathematically as a system of coupled nonlinear oscillators is traced back to [24]. Since then, a lot of researchers have tried to study the dynamics of the heartbeat based on limit cycle oscillators $[25,26]$. The model proposed in [27] describes the overall behavior of SA and AV nodes, captures the essential features of the cardiac conduction system, establishes a correspondence between system parameters and the physiological quantities, and is able to simulate different types of cardiac arrhythmias.

In this section, we apply the proposed adaptive output tracking controller to the four-dimensional coupled driven oscillators model in [27]. The model takes the following form:

$$
\begin{aligned}
& \dot{x}_{1}=\frac{1}{C_{1}} x_{2}, \\
& \dot{x}_{2}=-\frac{1}{L_{1}}\left[x_{1}+g\left(x_{2}\right)+R\left(x_{2}+x_{4}\right)\right]+A \cos (2 \pi \omega t) \\
& \dot{x}_{3}=\frac{1}{C_{2}} x_{4}, \\
& \dot{x}_{4}=-\frac{1}{L_{2}}\left[x_{3}+f\left(x_{4}\right)+R\left(x_{2}+x_{4}\right)\right],
\end{aligned}
$$

where $x_{2}$ and $x_{4}$ describe the action potential of the SA and AV nodes, and $x_{1}$ and $x_{3}$ are the voltage corresponding to $x_{2}$ and $x_{4}, C_{1}, C_{2}, L_{1}$, and $L_{2}$ are some constant parameters in the model, $R$ is the constant coupling parameter, $A$ and $\omega$ are the amplitude and frequency of the driven signal, which is used to model ectopic pace-makers in some region of the cardiac tissue, and $g$ and $f$ are some nonlinear functions of the following form:

$$
\begin{aligned}
& f\left(x_{4}\right)=-x_{4}+\frac{1}{3} x_{4}^{3}, \\
& h\left(x_{2}\right)= \begin{cases}-x_{2}^{2}-\frac{1}{4}, & \left|x_{2}\right|<\frac{1}{2}, \\
-x_{2}, & x_{2}>\frac{1}{2}, \\
x_{2}, & x_{2}<-\frac{1}{2},\end{cases} \\
& g\left(x_{2}\right)=-x_{2}+\frac{1}{3} h\left(x_{2}\right) .
\end{aligned}
$$

Systems (38a) to (38d) are in the form of system (1), with $z_{1}=x_{2}, \xi=\left[x_{1}, x_{3}, x_{4}\right]^{T}, \xi_{r}=\left[r_{1}, r_{3}, r_{4}\right]^{T}$, where $r_{1}, r_{3}$, and $r_{4}$ are equilibrium (invariant) trajectories for the zero dynamics. It is also assumed that the right-hand side of (38b) is unknown. It can be readily shown that this system meets Assumptions 1-3. (Just note that the tracking dynamics are linear in the output variable $x_{2}$, and that the resulting system 


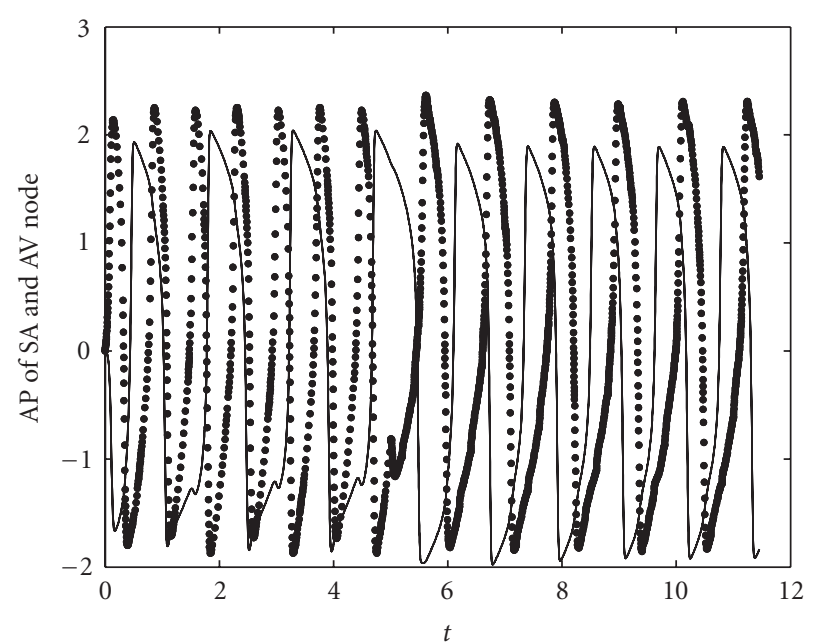

(a)

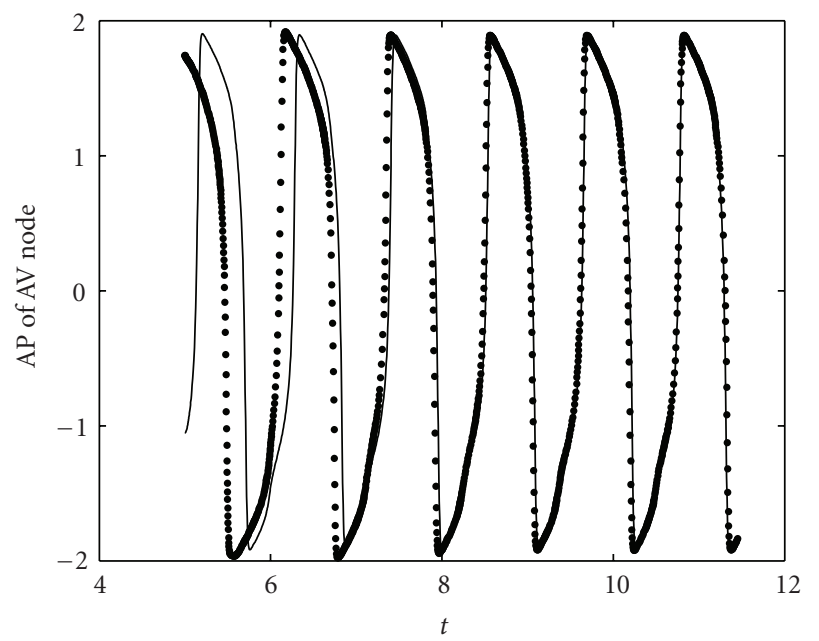

(c)

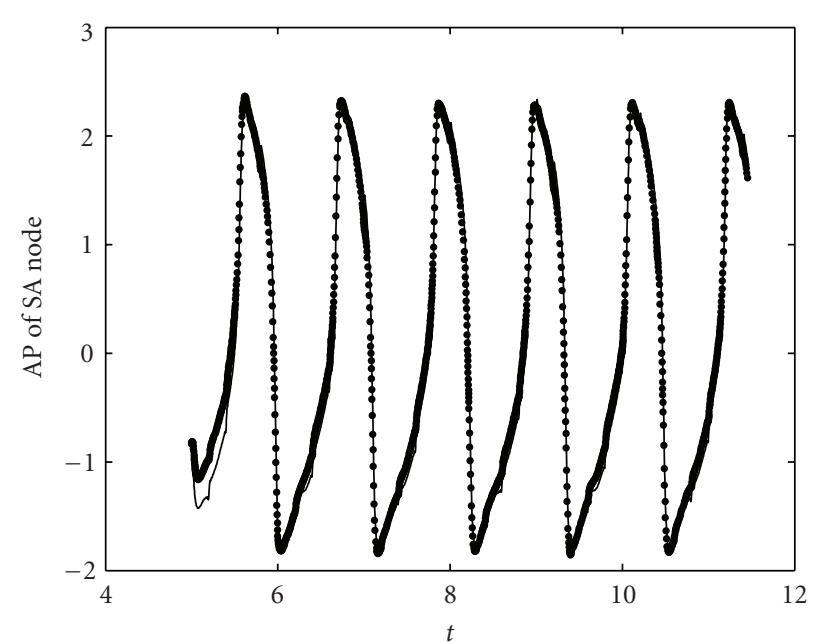

(b)

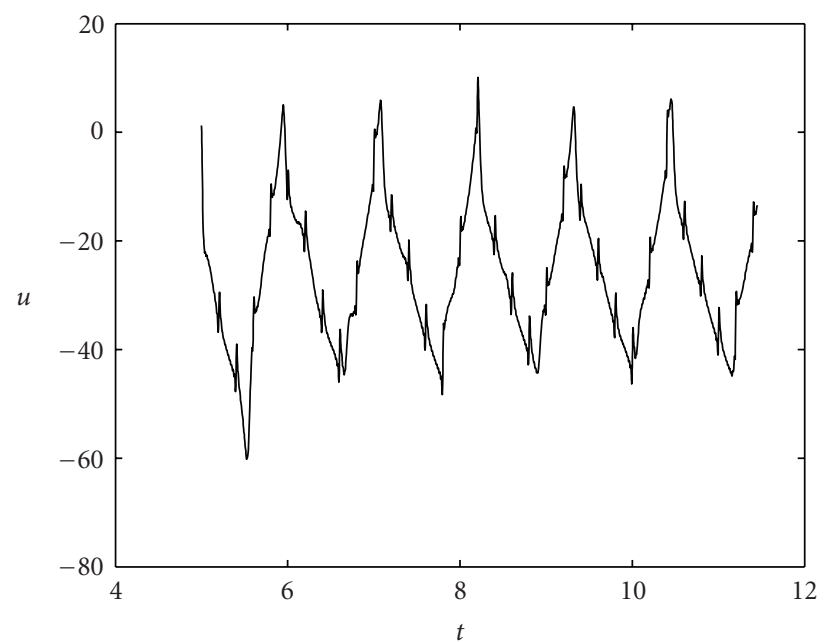

(d)

FIGURE 1: AV block suppression, tracking performances, and control action.

is stable for large values of $x_{4}$ and unstable in a small region containing the origin).

The electrical action potential can be measured by an transvenous electrode, which is a common part of artificial pacemakers. Given the following parameter values [27]: $C_{1}=$ $0.25 \mathrm{~F}, L_{1}=0.05 \mathrm{H}, C_{2}=0.675 \mathrm{~F}, L_{2}=0.027 \mathrm{H}$, and $R=0.11 \Omega$, the system exhibits a normal $1: 1$ rhythm. By setting $C_{1}=0.15 F$, one can generate an arrythmia of 2:1 AV block: for every two beats of the SA node, only one beat of AV node is observed. The control objective is to apply the proposed adaptive output tracking controller to make systems (38a) to (38d) track the normal 1:1 rhythm, in other words, to suppress the AV block arrythmia.

We choose to use a perturbation to the right-hand side of (38b) as the control

$$
\dot{x}_{2}=-\frac{1}{L_{1}}\left[x_{1}+g\left(x_{2}\right)+R\left(x_{2}+x_{4}\right)\right]+A \cos (2 \pi \omega t)+u .
$$

Physically, the control action is an electrical impulse sent to the heart through a transvenous electrode, which enters the system in an affine manner, as shown in (40).

Remark 2. Other potential control actuators are perturbation to the intrinsic frequency of the SA node (parameter $C_{1}$ ) and the coupling strength between the two nodes (parameter $R)$.

In this example, the unknown nonlinearity $\alpha\left(z, \xi_{r}\right)$ in (12) is as follows:

$$
\alpha\left(x_{2}, r_{1}, r_{4}\right)=\frac{1}{L_{1}}\left(r_{1}+g+R\left(x_{2}+r_{4}\right)\right)+A \cos (2 \pi \omega t) .
$$

For the simulation, the number of basis functions is $l=$ 11 , with $\sigma^{2}=5, \phi_{i}=i-6, i=1, \ldots, 11$, and $w_{m}=4$. The following tuning parameters are used, $k_{4}=k_{\mu}=k_{d}=k_{w}=$ $\gamma_{2}=25, \gamma_{w}=20$.

In the simulation, the controller is not turned on until $t=5$. Figure 1 shows the simulation results of the SA and 


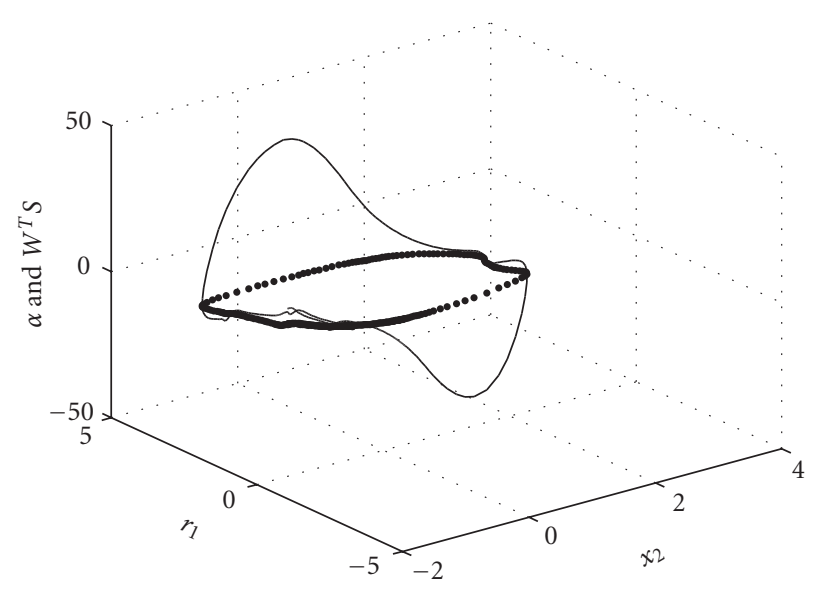

(a)

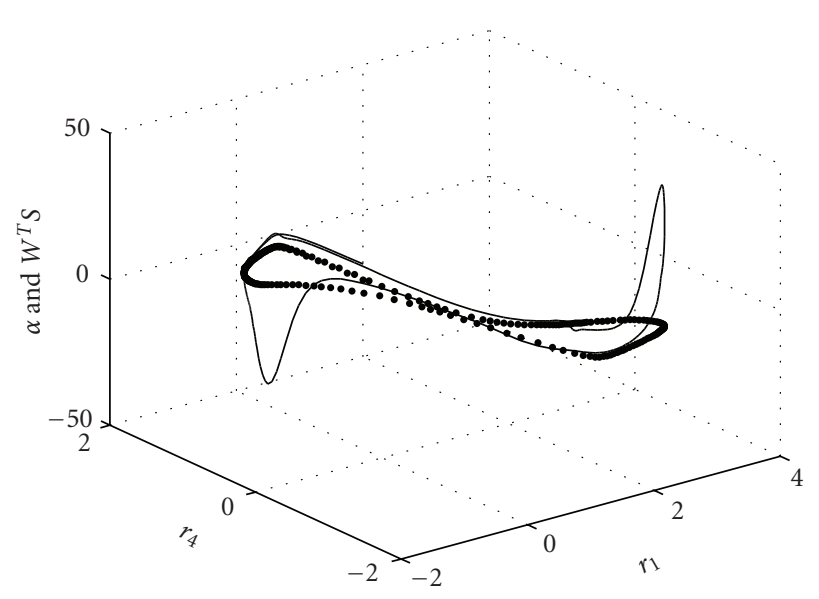

(c)

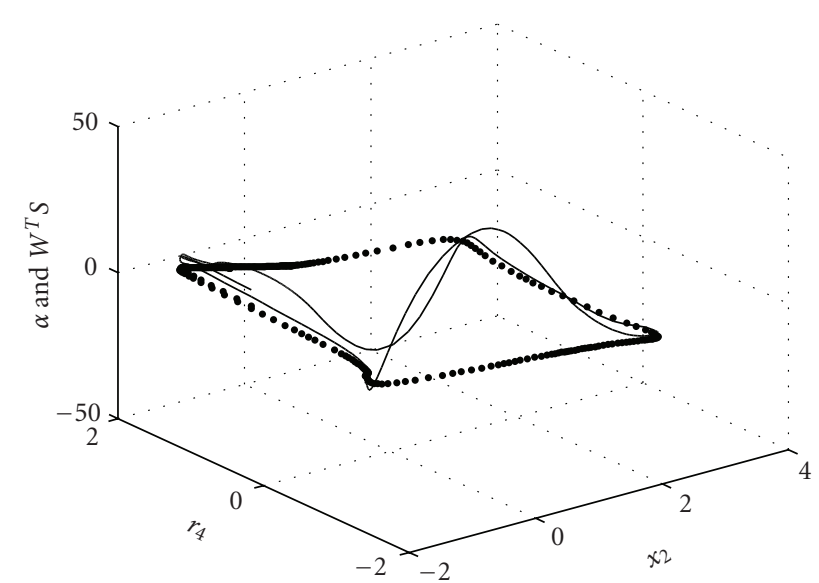

(b)

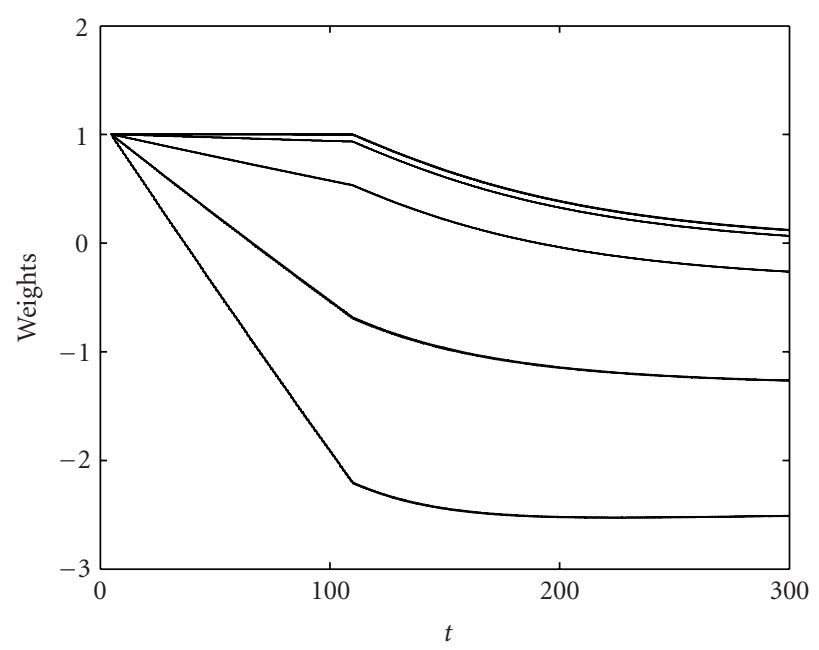

(d)

FIGURE 2: Unknown nonlinearities approximation and parameter convergence.

AV node rhythm, tracking performances, and the control action. In the subplot of SA and AV node action potential, the dotted line is the SA node action potential, and the solid line is the AV node action potential. It can be seen that before the controller is turned on, the rhythm is $2: 1$, with two beats of the SA node, only one beat of AV node is observed. After the controller is turned on at $t=5$, the rhythm is altered to 1:1 within one beat. The two subplots of tracking show that good tracking performances are achieved within a short time for the SA node and AV node, respectively. Figure 2 shows the approximation of the unknown nonlinearity and parameter estimation. The unknown nonlinearity $\alpha\left(x_{2}, r_{1}, r_{4}\right)$ is the solid line, and the approximation $\widehat{W}^{T} S\left(x_{2}, r_{1}, r_{4}\right)$ is the dotted line. After running the simulation for 300 seconds, good approximation is achieved along the $r_{1}$ and $r_{4}$ direction, and parameter estimations also converge.

\section{CONCLUSIONS}

In this research work, an adaptive output tracking controller is developed for a class of nonlinear systems with unknown nonlinearities, in order to address the heart dynamics control problem in a real-time framework. It is proved that the proposed controller is able to make the tracking error converges to a neighborhood of the origin exponentially fast. Simulation results show satisfactory performances that can be achieved when applying this technique to regulate irregular heart dynamics. In addition, good approximation of the unknown nonlinearities is also achieved by incorporating a persistent exciting signal in the parameter update law. The proposed technique is an alternative approach to the control of complex chaotic systems with unknown dynamics.

\section{APPENDIX}

Proof of Theorem 1. Consider the following Lyapunov function candidate for the $e_{c}$ subsystem (14):

$$
V_{1}=\frac{1}{2} e_{c}^{T} P e_{c}
$$

where $P$ is a symmetric positive definite function. 
The derivative of the Lyapunov function $V_{1}$ is given by

$$
\dot{V}_{1}=\frac{1}{2} e_{c}^{T}\left(P A_{c}+A_{c}^{T} P\right) e_{c}+e_{c}^{T} P B_{c} e_{\rho}
$$

Choosing $\Lambda=(1 / 2) B_{c}^{T} P$, we have

$$
e_{s}=e_{\rho}+\frac{1}{2} B_{c}^{T} P e_{c}
$$

A candidate Lyapunov function for the error dynamics is

$$
V_{2}=V_{1}+\frac{1}{2} \eta_{s}^{2}
$$

where $\eta_{s}=e_{s}+c^{T}(t) \widetilde{W}$, and $c^{T}(t)$ is the state of the filter (17) time-varying function to ensure persistency of excitation condition. Note that this filter is ISS with respect to the signal $B(t)=-b_{1} S\left(z, \xi_{r}\right)$ for any choice of gain $k_{t}$ large enough.

The time derivative of $V_{2}$ is given by

$$
\begin{aligned}
\dot{V}_{2}= & \dot{V}_{1}+\eta_{s}\left(\dot{e}_{s}+\dot{c}^{T}(t) \widetilde{W}+c^{T}(t) \dot{\widetilde{W}}\right) \\
= & \frac{1}{2} e_{c}^{T}\left(P A_{c}+A_{c}^{T} P\right) e_{c}+e_{c}^{T} P B_{c}\left(e_{s}-\frac{1}{2} B_{c}^{T} P e_{c}\right) \\
& +\eta_{s}\left(\dot{e}_{\rho}+\frac{1}{2} B_{c}^{T} P \dot{e}_{c}+\dot{c}^{T}(t) \widetilde{W}+c^{T}(t) \dot{\widetilde{W}}\right) .
\end{aligned}
$$

Substitution of $e_{\rho}=e_{s}-(1 / 2) B_{c}^{T} P e_{c}$ yields

$$
\begin{aligned}
\dot{V}_{2}= & \frac{1}{2} e_{c}^{T}\left(P A_{c}+A_{c}^{T} P\right) e_{c}-\frac{1}{2} e_{c}^{T} P B_{c} B_{c}^{T} P e_{c} \\
& +e_{c}^{T} P B_{c} e_{s}+\eta_{s}\left(-y_{r}^{(\rho)}+f(z, \xi, u)-f\left(z, \xi_{r}, u\right)\right) \\
& +\eta_{s}\left(b(z, u)\left(u-\alpha\left(z, \xi_{r}\right)\right)+\dot{c}^{T}(t) \widetilde{W}+c^{T}(t) \dot{\widetilde{W}}\right) \\
& +\eta_{s}\left(\frac{1}{2} B_{c}^{T} P A_{c} e_{c}+\frac{1}{2} B_{c}^{T} P B_{c} e_{s}-\frac{1}{4} B_{c}^{T} P B_{c} B_{c}^{T} P e_{c}\right)
\end{aligned}
$$

Given that $e_{s}=\eta_{s}-c(t)^{T} \widetilde{W}$, and

$$
\frac{1}{2} e_{s}^{2} \leq \eta_{s}^{2}+\widetilde{W}^{T} c(t) c^{T}(t) \widetilde{W},
$$

we have

$$
\begin{aligned}
\dot{V}_{2} \leq & \frac{1}{2} e_{c}^{T}\left(P A_{c}+A_{c}^{T} P\right) e_{c}-\frac{1}{2} e_{c}^{T} P B_{c} B_{c}^{T} P e_{c} \\
& +\frac{k_{1}}{2} e_{c}^{T} P B_{c} B_{c}^{T} P e_{c}+\frac{1}{k_{1}} \eta_{s}^{2}+\frac{1}{k_{1}} \widetilde{W}^{T} c(t) c^{T}(t) \widetilde{W} \\
& +\eta_{s}\left(-y_{r}^{(\rho)}+f(z, \xi, u)-f\left(z, \xi_{r}, u\right)\right) \\
& +\eta_{s}\left(b(z, u)\left(u-\alpha\left(z, \xi_{r}\right)\right)+\dot{c}^{T}(t) \widetilde{W}+c^{T}(t) \dot{\widetilde{W}}\right) \\
& +\frac{1}{2} \eta_{s} B_{c}^{T} P A_{c} e_{c}+\frac{1}{2} \eta_{s}^{2} B_{c}^{T} P B_{c} \\
& -\frac{1}{4} \eta_{s} B_{c}^{T} P B_{c} B_{c}^{T} P e_{c}-\frac{1}{2} \eta_{s} B_{c}^{T} P B_{c} c^{T}(t) \widetilde{W}
\end{aligned}
$$

or

$$
\begin{aligned}
\dot{V}_{2} \leq & \frac{1}{2} e_{c}^{T}\left(P A_{c}+A_{c}^{T} P\right) e_{c}+\gamma_{1} e_{c}^{T} P B_{c} B_{c}^{T} P e_{c} \\
& +\eta_{s}\left(-y_{r}^{(\rho)}+f(z, \xi, u)-f\left(z, \xi_{r}, u\right)\right) \\
& +\eta_{s}\left(b(z, u)\left(u-\alpha\left(z, \xi_{r}\right)\right)+\dot{c}^{T}(t) \widetilde{W}+c^{T}(t) \dot{\widetilde{W}}\right) \\
& +\frac{1}{k_{1}} \widetilde{W}^{T} c(t) c^{T}(t) \widetilde{W}-\eta_{s} c^{T}(t) \widetilde{W}\left(\frac{1}{2} B_{c}^{T} P B_{c}\right) \\
& +\gamma_{2} \eta_{s}^{2}+\frac{k_{2}}{4} e_{c}^{T} A_{c}^{T} A_{c} e_{c},
\end{aligned}
$$

where $k_{1}, k_{2}$, and $k_{3}$ are positive constants and

$$
\begin{aligned}
& \gamma_{1}=\left\{-\frac{1}{2}+\frac{k_{1}}{2}+\frac{k_{3}}{8}\right\}, \\
& \gamma_{2}=\left\{\frac{1}{k_{1}}+\frac{1}{4 k_{2}} B_{c}^{T} P P B_{c}+\frac{1}{2} B_{C}^{T} P B_{c}+\frac{1}{8 k_{3}} B_{c}^{T} P B_{c} B_{c}^{T} P B_{c}\right\} .
\end{aligned}
$$

The constants $k_{1}$ and $k_{3}$ are chosen such that $\gamma_{1}<0$. If the matrix $P$ is chosen as a positive definite solution of the Riccati-like equation

$$
P A_{c}+A_{c}^{T} P+2 \gamma_{1} P B_{c} B_{c}^{T} P+\frac{1}{2} k_{2} A_{c}^{T} A_{c}+Q=0
$$

for some positive definite symmetric matrix $Q$, then inequality (A.9) becomes

$$
\begin{aligned}
\dot{V}_{2} \leq & -\frac{1}{2} e_{c}^{T} Q e_{c}+\gamma_{2} \eta_{s}^{2} \\
& +\eta_{s}\left(b(z, u)\left(u-\alpha\left(z, \xi_{r}\right)\right)+\dot{c}^{T}(t) \widetilde{W}+c^{T}(t) \dot{\widetilde{W}}\right) \\
& +\frac{1}{k_{1}} \widetilde{W}^{T} c(t) c^{T}(t) \widetilde{W}-\eta_{s} c^{T}(t) \widetilde{W}\left(\frac{1}{2} B_{c}^{T} P B_{c}\right) \\
& +\eta_{s}\left(-y_{r}^{(\rho)}+f(z, \xi, u)-f\left(z, \xi_{r}, u\right)\right) .
\end{aligned}
$$

Substituting (12), inequality (A.12) becomes

$$
\begin{aligned}
\dot{V}_{2} \leq & -\frac{1}{2} e_{c}^{T} Q e_{c}+\gamma_{2} \eta_{s}^{2}+\eta_{s} \\
& \times\left(b(z, u)\left(u-\widehat{W}^{T} S\left(z, \xi_{r}\right)-\mu_{1}(t)\right)+\dot{c}^{T}(t) \widetilde{W}+c^{T}(t) \dot{\widetilde{W}}\right) \\
& +\frac{1}{k_{1}} \widetilde{W}^{T} c(t) c^{T}(t) \widetilde{W}-\eta_{s} c^{T}(t) \widetilde{W}\left(\frac{1}{2} B_{c}^{T} P B_{c}\right) \\
& -\eta_{s} y_{r}^{(\rho)}+\eta_{s} L_{1}\|\bar{\xi}\|+\eta_{s} b(z, u) \widetilde{W}^{T} S\left(z, \xi_{r}\right) \\
\leq & -\frac{1}{2} e_{c}^{T} Q e_{c}+\gamma_{2} \eta_{s}^{2}+\frac{k_{\mu}}{2} b(z, u)^{2} \eta_{s}^{2}+\frac{1}{2 k_{\mu}} \mu_{1}(x(t))^{2} \\
& +\frac{k_{d}}{2} \eta_{s}^{2}+\frac{1}{2 k_{d}}\left(y_{r}^{(\rho)}\right)^{2}+\eta_{s} L_{1}\|\bar{\xi}\|
\end{aligned}
$$




$$
\begin{aligned}
& +\eta_{s}\left(b(z, u)\left(u-\widehat{W}^{T} S\left(z, \xi_{r}\right)+\dot{c}^{T}(t) \widetilde{W}+c^{T}(t) \dot{\widetilde{W}}\right)\right. \\
& +\frac{1}{k_{1}} \widetilde{W}^{T} c(t) c^{T}(t) \widetilde{W}-\eta_{s} c^{T}(t) \widetilde{W}\left(\frac{1}{2} B_{c}^{T} P B_{c}\right) \\
& +\eta_{s} b(z, u) \widetilde{W}^{T} S\left(z, \xi_{r}\right),
\end{aligned}
$$

where $k_{\mu}$ and $k_{d}$ are positive constants.

Consider the control structure shown in (15), we choose the following controller:

$$
\begin{aligned}
u=\widehat{W}^{T} S\left(z, \xi_{r}\right)+e_{s}( & -k_{4}-\frac{1}{b_{0}} \gamma_{2}-\frac{k_{\mu}}{2} b_{1}(z)-\frac{k_{d}}{2} \frac{1}{b_{0}} \\
& \left.-\frac{k_{w}}{2} \frac{b_{1}(z)^{2}}{b_{0}} S\left(z, \xi_{r}\right)^{T} S\left(z, \xi_{r}\right)\right) \\
=\widehat{W}^{T} S\left(z, \xi_{r}\right)+\eta_{s}( & -k_{4}-\frac{1}{b_{0}} \gamma_{2}-\frac{k_{\mu}}{2} b_{1}(z)-\frac{k_{d}}{2} \frac{1}{b_{0}} \\
& \left.-\frac{k_{w}}{2} \frac{b_{1}(z)^{2}}{b_{0}} S\left(z, \xi_{r}\right)^{T} S\left(z, \xi_{r}\right)\right)
\end{aligned}
$$$$
-c^{T}(t) \widetilde{W} k_{t}
$$

where $k_{4}>0$ is a constant, $b_{0}$ and $b_{1}(z)$ are the lower and upper bound in the inequality (13), and $k_{t}$ is given by

$$
\begin{aligned}
k_{t}= & -k_{4}-\frac{1}{b_{0}} \gamma_{2}-\frac{k_{\mu}}{2} b_{1}(z)-\frac{k_{d}}{2} \frac{1}{b_{0}} \\
& -\frac{k_{w}}{2} \frac{b_{1}(z)^{2}}{b_{0}} S\left(z, \xi_{r}\right)^{T} S\left(z, \xi_{r}\right) .
\end{aligned}
$$

The above control action constitutes filtered tracking error and the approximated nonlinearity.

The weight $\widehat{W}$ satisfies $\|\widehat{W}\| \leq w_{m}$, where the upper bound, $w_{m}$, is guaranteed in the design of an adaptive law (16).

Substitution of the controller equation (A.14) in the inequality (A.13) gives

$$
\begin{aligned}
\dot{V}_{2} \leq & -\frac{1}{2} e_{c}^{T} Q e_{c}-k_{4} b \eta_{s}^{2}+\eta_{s}\left(b c^{T}(t) \widetilde{W} k_{t}+\dot{c}^{T}(t) \widetilde{W}+c^{T}(t) \dot{\widetilde{W}}\right) \\
& +\eta_{s} b(z, u) \widetilde{W}^{T} S\left(z, \xi_{r}\right)+\eta_{s} L_{1}\|\bar{\xi}\| \\
& +\frac{1}{k_{1}} \widetilde{W}^{T} c(t) c^{T}(t) \widetilde{W}-\eta_{s} c^{T}(t) \widetilde{W}\left(\frac{1}{2} B_{c}^{T} P B_{c}\right) \\
& +\frac{1}{2 k_{\mu}} \mu_{1}(x(t))^{2}+\frac{1}{2 k_{d}}\left(y_{r}^{(\rho)}\right)^{2} \\
& -\frac{k_{w}}{2} \frac{b_{1}(z)^{2}}{b_{0}} b S\left(z, \xi_{r}\right)^{T} S\left(z, \xi_{r}\right) \eta_{s}^{2},
\end{aligned}
$$

because of the inequality (13). and

The adaptive law (16) is chosen such that $\|\widehat{W}\| \leq w_{m}$,

$$
-\widetilde{W}^{T} c(t) e_{s}+\frac{1}{\gamma_{w}} \widetilde{W}^{T} \dot{\widehat{W}} \leq 0
$$

It takes the following form:

$$
\dot{\widehat{W}}=\left\{\begin{array}{cc}
\gamma_{w} c(t) e_{s}, & \text { if }\|\widehat{W}\|<w_{m}, \text { or } \\
& \|\widehat{W}\|=w_{m}, \text { and } \\
& \widehat{W}^{T} c(t) e_{s} \leq 0, \\
\gamma_{w} c(t) e_{s}-\gamma_{w} \frac{\widehat{W} \widehat{W}^{T} c(t) e_{s}}{\|\widehat{W}\|^{2}}, & \text { if }\|\widehat{W}\|=w_{m} \text { and } \\
& \widehat{W}^{T} c(t) e_{s}>0 .
\end{array}\right.
$$

Substitution of the controller equation (A.14), the parameter update law inequality (A.17), and the $c(t)$ dynamics (17), in the inequality (A.16), gives

$$
\begin{aligned}
\dot{V}_{2} \leq & -\frac{1}{2} e_{c}^{T} Q e_{c}-k_{4} b \eta_{s}^{2}+\gamma_{w} c^{T}(t) c(t) \eta_{s}^{2} \\
& +\frac{k_{w}}{2}\left(b-b_{1}\right)^{2} \eta_{s}^{2} S^{T} S-\frac{k_{w}}{2} \frac{b_{1}^{2}}{b_{0}} b S^{T} S \eta_{s}^{2} \\
& -\left(\left(b_{1}-b\right) k_{t}+\gamma_{w} c^{T}(t) c(t)\right) c^{T}(t) \widetilde{W} \eta_{s}+\eta_{s} L_{1}\|\bar{\xi}\| \\
& +\frac{1}{2 k_{\mu}} \mu_{1}(t)^{2}+\frac{1}{2 k_{d}}\left(y_{r}^{(\rho)}\right)^{2} \\
& +\frac{1}{2 k_{w}} \widetilde{W}^{T} \widetilde{W}+\frac{1}{k_{1}} \widetilde{W}^{T} c(t) c^{T}(t) \widetilde{W},
\end{aligned}
$$

where $b_{1}=b_{1}(z) b=b(z, u)$, and $S=S\left(z, \xi_{r}\right)$ due to space limits.

Since $\left(b(z, u) / b_{1}(z)-1\right)^{2} \leq 1$, it follows that

$$
\begin{aligned}
\dot{V}_{2} \leq & -\frac{1}{2} e_{c}^{T} Q e_{c}-k_{4} b \eta_{s}^{2}+\gamma_{w} c^{T}(t) c(t) \eta_{s}^{2} \\
& -\left(\left(b_{1}-b\right) k_{t}+\gamma_{w} c^{T}(t) c(t)\right) c^{T}(t) \widetilde{W} \eta_{s} \\
& +\eta_{s} L_{1}\|\bar{\xi}\|+\frac{1}{2 k_{\mu}} \mu_{1}(t)^{2}+\frac{1}{2 k_{d}}\left(y_{r}^{(\rho)}\right)^{2} \\
& +\frac{1}{2 k_{w}} \widetilde{W}^{T} \widetilde{W}+\frac{1}{k_{1}} \widetilde{W}^{T} c(t) c^{T}(t) \widetilde{W} .
\end{aligned}
$$

Let $V=V_{2}+(1 / 2) \widetilde{W}^{T} \widetilde{W}$, and let $k_{c}=\left(b_{1}-b\right) k_{t}$, we have

$$
\begin{aligned}
\dot{V} \leq & -\frac{1}{2} e_{c}^{T} Q e_{c}-k_{4} b \eta_{s}^{2} \\
& -\left(\gamma_{w}+\frac{k_{w}}{2}\left(k_{c}+\gamma_{w} c^{T}(t) c(t)-\gamma_{w}\right)^{2}\right) c^{T}(t) c(t) \eta_{s}^{2} \\
& +\left(\frac{1}{k_{1}}-\gamma_{w}\right) \widetilde{W}^{T} c(t) c^{T}(t) \widetilde{W}+\frac{1}{2 k_{w}} \widetilde{W}^{T} \widetilde{W} \\
& +\eta_{s} L_{1}\|\bar{\xi}\|+\frac{1}{2 k_{\mu}} \mu_{1}(x(t))^{2}+\frac{1}{2 k_{d}}\left(y_{r}^{(\rho)}\right)^{2} .
\end{aligned}
$$


Let $\tilde{k}_{4}=k_{4} b_{0}-\left(\gamma_{w}+\left(k_{w} / 2\right)\left(k_{c}+\gamma_{w} c^{T}(t) c(t)-\right.\right.$ $\left.\left.\gamma_{w}\right)^{2}\right) c^{T}(t) c(t)>0$, inequality (A.21) becomes

$$
\begin{aligned}
\dot{V} \leq & -\frac{1}{2} e_{c}^{T} Q e_{c}-\widetilde{k}_{4} \eta_{s}^{2}+\left(\frac{1}{k_{1}}-\gamma_{w}\right) \widetilde{W}^{T} c(t) c^{T}(t) \widetilde{W} \\
& +\frac{1}{k_{w}} \widetilde{W}^{T} \widetilde{W}+\eta_{s} L_{1}\|\bar{\xi}\|+\frac{1}{2 k_{\mu}} \mu_{1}(x(t))^{2}+\frac{1}{2 k_{d}}\left(y_{r}^{(\rho)}\right)^{2} .
\end{aligned}
$$

Noting that, by assumption, $\left|\mu_{l}(x(t))\right| \leq \bar{\mu}_{l},\left\|y_{r}^{(\rho)}\right\| \leq \bar{\nu}_{1}$, and using the fact that

$$
\begin{aligned}
\widetilde{W}^{T} \widetilde{W} & =\left(\widehat{W}-W^{*}\right)^{T}\left(\widehat{W}-W^{*}\right) \\
& \leq\|\widehat{W}\|^{2}+2\left|\widehat{W}^{T} W^{*}\right|+\left\|W^{*}\right\|^{2} \leq 4 w_{m}^{2},
\end{aligned}
$$

we obtain

$$
\begin{aligned}
\dot{V} \leq & -\frac{1}{2} e_{c}^{T} Q e_{c}-\widetilde{k}_{4} \eta_{s}^{2}+\eta_{s}\left(L_{1}\|\bar{\xi}\|\right)+\frac{1}{2 k_{d}} \bar{v}_{1}^{2}+\frac{1}{2 k_{\mu}} \bar{\mu}_{l}^{2} \\
& +\frac{1}{k_{w}} 4 w_{m}^{2}+\left(\frac{1}{k_{1}}-\gamma_{w}\right) \widetilde{W}^{T} c(t) c^{T}(t) \widetilde{W} .
\end{aligned}
$$

Consider the following composite Lyapunov function for the closed-loop system (15), (16), (6), and (7):

$$
V_{c}=V+\alpha U
$$

where $\alpha$ is a positive design parameter. The derivative of the Lyapunov function $V_{c}$ is given by

$$
\begin{aligned}
\dot{V}_{c}= & \dot{V}+\alpha \dot{U} \\
\leq & -\frac{1}{2} e_{c}^{T} Q e_{c}-\widetilde{k}_{4} \eta_{s}^{2}+\frac{k_{5}}{2} \eta_{s}^{2}+\frac{1}{2 k_{5}} L_{1}^{2}\|\bar{\xi}\|^{2}-c_{3} \alpha\|\bar{\xi}\|^{2} \\
& +\Gamma \frac{k_{6}}{2} e_{c}^{T} e_{c}+\Gamma \frac{k_{6}}{2} e_{s}^{2}+\frac{1}{2 k_{6}} c_{4}^{2} \alpha^{2}\|\bar{\xi}\|^{2}+\frac{1}{2 k_{d}} \bar{\nu}_{1}^{2}+\frac{1}{2 k_{\mu}} \bar{\mu}_{l}^{2} \\
& +\frac{1}{k_{w}} 4 w_{m}^{2}+\left(\frac{1}{k_{1}}-\gamma_{w}\right) \widetilde{W}^{T} c(t) c^{T}(t) \widetilde{W}
\end{aligned}
$$

where $\Gamma=\left(1+B_{c}^{T} P P B_{c}\right)$, since, by definition, the trajectory error vector is such that

$$
e^{T} e=e_{c}^{T} e_{c}+e_{n}^{2} \leq\left(1+B_{c}^{T} P P B_{c}\right) e_{c}^{T} e_{c}+e_{s}^{2},
$$

or

$$
e^{T} e \leq\left(1+B_{c}^{T} P P B_{c}\right)\left(e_{c}^{T} e_{c}+e_{s}^{2}\right)
$$

Define

$$
\begin{gathered}
\lambda=\min \left\{\frac{\lambda_{\min }\{Q\}}{2 \lambda_{\max }\{P\}}-\Gamma \frac{k_{6}}{2}, \tilde{k}_{4}-\frac{k_{5}}{2}-\Gamma \frac{k_{6}}{2},\right. \\
\left.c_{3}-\frac{L_{1}^{2}}{2 k_{5} \alpha c_{2}}-\frac{c_{4}^{2} \alpha}{2 k_{6} c_{2}}\right\},
\end{gathered}
$$

$\Gamma k_{6}+\left(1 / k_{1}\right)+\lambda<\gamma_{w}$, and the gains of the two interconnected system (6) and (7) are such that $c_{3}-L_{1}^{2} / 2 k_{5} \alpha c_{2}-c_{4}^{2} \alpha / 2 k_{6} c_{2}>$ 0 , we have

$$
\begin{aligned}
\dot{V}_{c} \leq & -\lambda e_{c} P e_{c}-\lambda \eta_{s}^{2}-\lambda \alpha U-\lambda \widetilde{W}^{T} c(t) c^{T}(t) \widetilde{W} \\
& +\frac{1}{2 k_{d}} \bar{v}_{1}^{2}+\frac{1}{2 k_{\mu}} \bar{\mu}_{l}^{2}+\frac{1}{k_{w}} 4 w_{m}^{2} \\
\leq & -\frac{\lambda}{2} e_{c} P e_{c}-\frac{\lambda}{2} e_{s}^{2}-\frac{\lambda}{2} \alpha U+\frac{1}{2 k_{d}} \bar{v}_{1}^{2}+\frac{1}{2 k_{\mu}} \bar{\mu}_{l}^{2}+\frac{1}{k_{w}} 4 w_{m}^{2},
\end{aligned}
$$

or

$$
\dot{V}_{c} \leq-\lambda V_{c}+\frac{1}{2 k_{d}} \bar{v}_{1}^{2}+\frac{1}{2 k_{\mu}} \bar{\mu}_{l}^{2}+\frac{1}{k_{w}} 4 w_{m}^{2} .
$$

It follows that the error vector, $e$, variable $\eta_{s}$, and the parameter estimation errors, $\widetilde{W}$, are bounded. Since the tracking trajectory $y_{r}$ and its first $\rho$ derivatives are bounded, it follows that the states $z$ and $\xi$ are both bounded.

Integration of inequality (A.31) yields an explicit bound for $\left\|\eta_{s}\right\|$ given by

$$
\left\|\eta_{s}\right\| \leq \alpha_{\eta} e^{-\lambda_{\eta}\left(t-t_{0}\right)}+\frac{1}{\sqrt{k_{\eta}}} \sqrt{\bar{\mu}_{l}^{2}+\bar{\nu}_{1}^{2}+w_{m}^{2}},
$$

where $\alpha_{\eta}=\sqrt{2 V_{c}(0)}, \lambda_{\eta}=\lambda / 2$, and $k_{\eta}=\min \left[2 k_{d}, 2 k_{\mu}, k_{w} / 4\right]$.

Next, we derive a persistency of excitation condition that guarantees the convergence of the parameter estimates to the ideal weights, $W^{*}$.

A solution to (17) is given by

$$
c^{T}(t)=e^{-K(t)\left(t-t_{0}\right)} c^{T}\left(t_{0}\right)+\int_{t_{0}}^{t} e^{-K(t)(t-\tau)} B(\tau) d \tau .
$$

It is obvious that the differential equation

$$
\dot{c}^{T}(t)=-K(t) c^{T}(t)
$$

is globally exponentially stable.

The element $B(t)$ is a bounded function of time. Note that

$$
\|B(t)\|^{2}=b_{1}(z)^{2} S^{T} S .
$$

For the particular choice of basis functions proposed in this paper, we have $\|S\| \leq \sqrt{N}$, where $N$ is the number of weights used in the approximation. The boundedness of $b_{1}(z)$ is obvious, since $z$ is bounded. Therefore, it follows that the norm of $B(t)$ is bounded by some positive number $B_{M}$, that is,

$$
\|B(t)\| \leq B_{M} .
$$

Using the exponential stability of system (A.34) and the bound on $B(t)$, an explicit bound for the solution of (17) can be obtained as follows:

$$
\left\|c^{T}(t)\right\| \leq C e^{-\lambda_{c}\left(t-t_{0}\right)}+C \frac{B_{M}}{\lambda_{c}}
$$

where $C=\left\|c^{T}\left(t_{0}\right)\right\|>0$ and $\lambda_{c}>0$ is a positive constant. 
Next, it is shown that the parameter estimation error $\widetilde{W}$ converges to a neighborhood of the origin. In the following, it is shown that, under Assumption 4, the unperturbed (i.e., $\left.\eta_{s} \equiv 0\right)$ dynamics of the parameter estimation errors are exponentially stable.

Lemma 1 establishes that the origin of the differential equation

$$
\dot{\widetilde{W}}=-\gamma_{w} c(t) c^{T}(t) \widetilde{W}
$$

is an exponentially stable equilibrium. In fact, it follows from the proof of Lemma 1 that the Lyapunov function $V_{w}=$ $\left(1 / 2 \gamma_{w}\right) \widetilde{W}^{T} \widetilde{W}$ is such that

$$
\dot{V}_{w}=-\widetilde{W}^{T} c(t) c^{T}(t) \widetilde{W} \leq-c_{w}\|\widetilde{W}\|^{2}
$$

for $c_{w}>0$ a positive constant.

It follows from the property of the projection algorithm that the rate of change of $V_{w}$ along the trajectories of (A.18) is given by

$$
\dot{V}_{w} \leq-\widetilde{W}^{T} c(t) c^{T}(t) \widetilde{W}-\widetilde{W}^{T} c(t) \eta_{s} .
$$

Completing the squares, we get

$$
\dot{V}_{w} \leq-\frac{1}{2} \widetilde{W}^{T} c(t) c^{T}(t) \widetilde{W}+\frac{1}{2} \eta_{s}^{2} .
$$

Substitution of (A.32) yields

$$
\dot{V}_{w} \leq-c_{w} \gamma_{w} V_{w}+\alpha_{\eta}^{2} e^{-2 \lambda_{\eta}\left(t-t_{0}\right)}+\frac{1}{k_{\eta}}\left(\bar{\mu}_{l}^{2}+\bar{\nu}_{1}^{2}+w_{m}^{2}\right) .
$$

By integrating, we get

$$
\begin{aligned}
V_{w} \leq & \max \left\{V_{w}\left(t_{0}\right),\left|\frac{\alpha_{\eta}^{2}}{c_{w} \gamma_{w}-2 \lambda_{\eta}}\right|, \frac{1}{c_{w} \gamma_{w} k_{\eta}}\left(\bar{\mu}_{l}^{2}+\bar{\nu}_{1}^{2}+w_{m}^{2}\right)\right\} \\
& \times \exp \left[-\min \left\{c_{w} \gamma_{w}, 2 \lambda_{\eta}\right\}\left(t-t_{0}\right)\right] \\
& +\frac{1}{c_{w} \gamma_{w} k_{\eta}}\left(\bar{\mu}_{l}^{2}+\bar{\nu}_{1}^{2}+w_{m}^{2}\right) .
\end{aligned}
$$

Consequently, the parameter estimation error is guaranteed to decay exponentially as

$$
\|\widetilde{W}\| \leq \alpha_{w} e^{-\lambda_{w}}\left(t-t_{0}\right)+\sqrt{\frac{2}{k_{\eta} c_{w}}}\left(\bar{\mu}_{l}^{2}+\bar{\nu}_{1}^{2}+w_{m}^{2}\right)^{1 / 2} .
$$

Taking the limit as $t \rightarrow \infty$ confirms that the estimation error converges to a small adjustable neighborhood of the origin given by

$$
\lim _{t \rightarrow \infty}\|\widetilde{W}\| \leq \sqrt{\frac{2}{k_{\eta} c_{w}}}\left(\bar{\mu}_{l}^{2}+\bar{\nu}_{1}^{2}+w_{m}^{2}\right)^{1 / 2} .
$$

Under the assumption that the persistency of excitation condition is fulfilled, we have demonstrated that the parameter estimation error and the redefined state variable, $\eta_{s}$, converge exponentially fast to an adjustable neighborhood of the origin. The size of the neighborhood can be changed by increasing the size of the controller gain and by reducing the size of the approximation error.

Inequality (23) can be deduced from inequality (A.30) as follows. Inequality (A.30) can be written as

$$
\begin{aligned}
\dot{V}_{c} \leq & -\frac{1}{2} \lambda_{\min }\{P\} e_{c}^{T} e_{c}-\frac{\lambda}{2} e_{s}^{2}-\frac{\lambda}{2} \alpha U+\frac{1}{2 k_{d}} \bar{\nu}_{1}^{2} \\
& +\frac{1}{2 k_{\mu}} \bar{\mu}_{l}^{2}+\frac{1}{k_{w}} 4 w_{m}^{2},
\end{aligned}
$$

where $\lambda_{\min }\{Q\}$ is the smallest eigenvalue of $Q$. Integration of inequality (A.46) gives

$$
\begin{aligned}
V_{c}(t)- & V_{c}(0) \\
\leq & -\int_{0}^{t}\left(\frac{1}{2} \lambda_{\min }\{P\} e_{c}(\sigma)^{T} e_{c}(\sigma)+\frac{\lambda}{2} e_{s}(\sigma)^{2}+\frac{\lambda}{2} \alpha U(\sigma)^{2}\right) d \sigma \\
& +\frac{t}{2}\left(\frac{1}{2 k_{d}} \bar{\nu}_{1}^{2}+\frac{1}{2 k_{\mu}} \bar{\mu}_{l}^{2}+\frac{1}{k_{w}} 4 w_{m}^{2}\right) .
\end{aligned}
$$

This result implies that the following inequality holds:

$$
\frac{1}{t} \int_{0}^{t} e_{1}(\sigma)^{2} d \sigma \leq \frac{2 V_{c}(0)}{t \lambda_{\min }\{P\}}+\frac{1}{k}\left(\bar{\mu}_{l}^{2}+\bar{\nu}_{1}^{2}+w_{m}^{2}\right),
$$

where $k=\left(1 / \lambda_{\min }\{Q\}\right) \min \left\{k_{\mu}, k_{d}, k_{w} / 8\right\}$. Hence, the boundedness of the mean square error stated in inequality (23) is achieved with $\alpha_{0}=2 / \lambda_{\min }\{P\}$ as required.

Integration of inequality (A.31) gives

$$
\begin{aligned}
V_{c}(t) \leq & V_{c}(0) e^{-\lambda\left(t-t_{0}\right)}+\left(\frac{1}{2 k_{d}} \bar{\nu}_{1}^{2}+\frac{1}{2 k_{\mu}} \bar{\mu}_{l}^{2}+\frac{1}{k_{w}} 4 w_{m}^{2}\right) \\
& \times \int_{t_{0}}^{t} e^{-\lambda(t-\tau)} d \tau \\
\leq & V_{c}(0) e^{-\lambda\left(t-t_{0}\right)}+\left(\frac{1}{2 k_{d}} \bar{v}_{1}^{2}+\frac{1}{2 k_{\mu}} \bar{\mu}_{l}^{2}+\frac{1}{k_{w}} 4 w_{m}^{2}\right) .
\end{aligned}
$$

Given (A.7), we have

$$
\frac{1}{2 p_{c}} e_{s}^{2} \leq \eta_{s}^{2}+\|\widetilde{W}\|^{2},
$$

where $p_{c}=\sup _{t>0}\|c(t)\|^{2}$. With the positive constant

$$
p_{m}=\min \left\{\lambda_{\min }\{P\}, \frac{1}{2 p_{c}}\right\},
$$

inequality (A.49) simplifies to

$\frac{1}{2} e_{c}^{T} e_{c}+\frac{1}{2} e_{s}^{2} \leq \frac{V_{c}(0)}{p_{m}} e^{-\lambda\left(t-t_{0}\right)}+\frac{1}{p_{m}}\left(\frac{1}{2 k_{d}} \bar{\nu}_{1}^{2}+\frac{1}{2 k_{\mu}} \bar{\mu}_{l}^{2}+\frac{1}{k_{w}} 4 w_{m}^{2}\right)$.

It follows from inequality (A.52) that

$$
e^{T} e \leq \Gamma \frac{V_{c}(0)}{p_{m}} e^{-\lambda\left(t-t_{0}\right)}+\Gamma \frac{1}{p_{m}}\left(\frac{1}{2 k_{d}} \bar{\nu}_{1}^{2}+\frac{1}{2 k_{\mu}} \bar{\mu}_{l}^{2}+\frac{1}{k_{w}} 4 w_{m}^{2}\right),
$$


where $\Gamma=2\left(1+B_{c}^{T} P P B_{c}\right)$. As a result, the adaptive learning tracking control guarantees that the tracking error, $e$, fulfills the following inequality:

$$
\|e\| \leq \alpha_{1} e^{-\beta_{1}\left(t-t_{0}\right)}+\alpha_{2} \frac{1}{\sqrt{k}}\left(\bar{\mu}_{l}+\bar{\nu}_{1}+w_{m}\right),
$$

where

$$
\begin{gathered}
\alpha_{1}=\left(\Gamma \frac{V(0)}{p_{m}}\right)^{1 / 2}, \\
\beta_{1}=\lambda, \\
\alpha_{2}=\left(\frac{\Gamma}{p_{m}}\right)^{1 / 2}, \\
k=\min \left[2 k_{d}, 2 k_{\mu}, \frac{k_{w}}{4}\right] .
\end{gathered}
$$

This completes the proof.

Proof of Theorem 2. We consider the Lyapunov function $V_{2}=(1 / 2) e_{c}^{T} P e_{c}+(1 / 2) e_{s}^{2}+\left(1 / 2 \gamma_{w}\right) \widetilde{W}^{T} \widetilde{W}$. We first note that $\hat{e}=e-D(\epsilon) \eta$, where $D(\epsilon)=\operatorname{diag}\left[\epsilon^{n-1}, \ldots, 1\right]$ and write the closed-loop system as follows:

$$
\begin{aligned}
& \dot{e}=A_{0} e+B_{0}\left(f\left(x, \Psi_{s}\left(e-D(\epsilon) \eta, \widehat{W}, Y_{d}\right)-y_{d}^{(n)}\right),\right.
\end{aligned}
$$

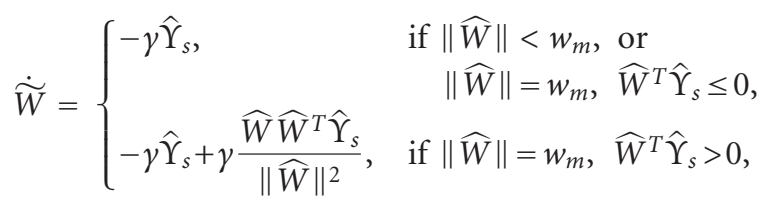

$$
\begin{aligned}
& \epsilon \dot{\eta}=A_{0} \eta+\epsilon B_{0}\left(f\left(x, \Psi_{s}\left(e-D(\epsilon) \eta, \widehat{W}, Y_{d}\right)\right)-y_{d}^{(n)}\right) .
\end{aligned}
$$

By Lipschitz continuity of the projection algorithm [28], it follows that for a sufficiently small $\epsilon$ the inequality

$\left\|\operatorname{Proj}\left(\Upsilon_{s}\left(e-D(\epsilon) \eta, Y_{d}\right), \widehat{W}\right)-\operatorname{Proj}\left(\Upsilon_{s}\left(e, Y_{d}\right), \widehat{W}\right)\right\| \leq \gamma L_{1}\|\eta\|$

holds on $\Omega$ for some positive nonzero constant $L_{1}$ and all $0<\epsilon<\epsilon_{1}$.

Similarly, it follows from the continuous differentiability of $b_{1}(x)$ and $S(x)$ that the following

$$
\left\|\Psi_{s}\left(e-D(\epsilon) \eta, Y_{d}, \widehat{W}\right)-\Psi_{s}\left(e, Y_{d}, \widehat{W}\right)\right\| \leq L_{2}\|\eta\|
$$

holds on $\Omega$ for some positive nonzero constant $L_{2}$ and all $0<\epsilon \leq \epsilon_{2}$.

Using (A.31) and (A.42), the derivative of the Lyapunov function $V_{2}$ along the trajectories of the closed-loop system is such that

$$
\begin{aligned}
\dot{V}_{2} \leq & -k_{5} V_{2}+c_{2}+e_{s} b(x, u) \\
\times & \left(\Psi_{s}\left(e-D(\epsilon) \eta, Y_{d}, \widehat{W}\right)-\Psi_{s}\left(e, Y_{d}, \widehat{W}\right)\right) \\
+ & \frac{1}{\gamma} \widetilde{W}^{T}\left(\operatorname{Proj}\left(\Upsilon_{s}\left(e-D(\epsilon) \eta, Y_{d}\right), \widehat{W}\right)\right. \\
& \left.-\operatorname{Proj}\left(\Upsilon_{s}\left(e, Y_{d}\right), \widehat{W}\right)\right),
\end{aligned}
$$

where $k_{5}=\lambda / 2, c_{2}$ is a positive constant arising from the constant terms in (A.31) and (A.42). In the light of the Lipschitz inequalities (A.57) and (A.58), we obtain

$$
\begin{aligned}
\dot{V}_{2} & \leq-k_{5} V_{2}+c_{2}+L_{2} L_{3} \bar{b}\|\eta\|+2 w_{m} L_{1}\|\eta\| \\
& =-k_{5} V_{2}+c_{2}+k_{7}\|\eta\|,
\end{aligned}
$$

where $\bar{b}=\max _{e \in \Omega, Y_{d} \in Y} b_{1}\left(e, Y_{d}\right)$, and $L_{3}$ is the maximum of $e_{s}$ on $\Omega$. Similarly, the derivative of $W(\eta)$ along the trajectories of the closed-loop system is given by

$$
\begin{aligned}
& \epsilon \dot{W} \leq-\eta^{T} \eta+2 \epsilon \eta^{T} P_{0} B_{0} \\
& \times\left[b ( x , u ) \left(\Psi_{s}\left(e-D(\epsilon) \eta, Y_{d}, \widehat{W}\right)\right.\right. \\
&\left.\left.\quad-\left(W^{*} S\left(e+Y_{d}\right)-\mu_{l}(x(t))\right)\right)-y_{d}^{(n)}\right] .
\end{aligned}
$$

Since $\left|\Psi_{s}\right| \leq S_{u},\left\|W^{*}\right\| \leq w_{m},\left\|S\left(e+Y_{d}\right)\right\| \leq \bar{S}$, and $\mu_{l}(x(t)) \leq \bar{\mu}_{l}$, we obtain

$$
\begin{aligned}
\epsilon \dot{W} & \leq-\eta^{T} \eta+2 \epsilon\|\eta\| \lambda_{\max }\left(P_{0}\right)\left(\bar{b}\left(S_{u}+2 w_{m} \bar{S}+\bar{\mu}_{l}\right)+\bar{\nu}_{1}\right) \\
& =-\eta^{T} \eta+2 \epsilon\|\eta\| \lambda_{\max }\left(P_{0}\right) k_{6} .
\end{aligned}
$$

Then, it follows that $\dot{V}_{2} \leq 0$ on $\left\{V_{2}=c_{1}\right\} \times\left\{W(\eta) \leq \rho \epsilon^{2}\right\}$ as long as $\epsilon \leq\left(k_{5} c_{1}-c_{2}\right) / k_{7} \sqrt{\rho / \lambda_{\min }\left(P_{0}\right)}=\epsilon_{3}<\min \left\{\epsilon_{1}, \epsilon_{2}\right\}$. Similarly, $\dot{W} \leq 0$ on $\left\{V_{2} \leq c_{1}\right\} \times\left\{W(\eta)=\rho \epsilon^{2}\right\}$ if $\rho \leq$ $4 \lambda_{\max }\left(P_{0}\right)^{3} k_{6}^{2}$. As a result, we show that the set $\left\{V_{2} \leq c_{1}\right\} \times$ $\left\{W(\eta) \leq \rho \epsilon^{2}\right\}$ is positively invariant for all $0<\epsilon \leq \epsilon_{3}$.

Consider initial conditions $\{e(0), \widetilde{W}(0)\} \in S$, and $\{\hat{e}(0)\} \in O$ where $S$ is a compact subset $\Omega_{b} \subset \Omega$ and $O$ is a compact subset of $\mathbb{R}^{n}$. It follows that $\eta(0) \leq k_{4} / \epsilon^{(n-1)}$, where $k_{4}$ is a positive constant which is related to the size of the compact sets $S$ and $O$.

Note that $f\left(e, Y_{d}, \widetilde{W}, \mu\right)=\left[e_{2}, e_{3}, \ldots, b(x, u)\left(\Psi_{s}(\cdot)-\right.\right.$ $\left.W^{* T} S\left(e+Y_{d}\right)-\mu_{l}(x(t))\right]^{T}$ is bounded on $\Omega$. That is, $\left\|f\left(x, \Psi_{s}(\cdot)\right)\right\| \leq k_{5}$. By construction, the adaptive learning rate is such that $\left\|\operatorname{Proj}\left(\Upsilon_{s}(\cdot), \widehat{W}\right)\right\| \leq k_{6}$. As a result, the closed-loop trajectories of the system starting in $S$ are such that

$$
\begin{gathered}
\|e(t)-e(0)\| \leq k_{5} t, \\
\|\widetilde{W}(t)-\widetilde{W}(0)\| \leq k_{6} t .
\end{gathered}
$$

Therefore, there is a time $T_{e}$ at which the closed-loop trajectory $(e(t), \widetilde{W}(t))$ escapes the set $\Omega$.

From (A.62), it follows that

$$
\dot{W} \leq-\frac{1}{2 \epsilon} \eta^{T} \eta
$$

if $W \geq \rho \epsilon^{2}$. Therefore, we have that

$$
\dot{W} \leq-\frac{1}{2 \epsilon \lambda_{\max }\left(P_{0}\right)} W
$$


or

$$
W(t)=e^{-\left(1 / 2 \epsilon \lambda_{\max }\right)\left(P_{0}\right) t} W(0),
$$

for $W \geq \rho \epsilon^{2}$. As a result, we can find an $\epsilon<\epsilon_{3}$ and a $T(\epsilon)>0$ such that $W(t) \leq \rho \epsilon^{2}, \forall t \geq T(\epsilon)$. Moreover, it is always possible to pick $\epsilon_{3}$ small enough such that $T\left(\epsilon_{3}\right) \leq T_{0}$. As a result, picking $\epsilon_{1}^{*}=\min \left\{\epsilon_{1}, \epsilon_{2}, \epsilon_{3}\right\}$ ensures that the trajectories of the closed-loop process starting in $S \times O$ enter the compact set $\Lambda=\left\{V_{2} \leq c_{1}\right\} \times\left\{W \leq \rho \epsilon^{2}\right\}$ in finite time $T(\epsilon) \forall 0<\epsilon \leq \epsilon_{1}^{*}$.

On the set $\Lambda$, it is shown that

$$
\dot{V}_{2} \leq-k_{1} V_{2}+c_{2}+k_{3} \sqrt{\frac{\rho}{\lambda_{\min }}} \epsilon .
$$

Hence, we have

$$
\dot{V}_{2} \leq-\frac{k_{1}}{2} V_{2}
$$

when $\left\{V_{2} \geq 2 c_{2}+2 k_{3} \sqrt{\left(\rho / \lambda_{\min }\right)} \epsilon\right\}$ and, therefore, the trajectories of the system must be such that $\lim _{t \rightarrow \infty} V_{2}(t) \leq$ $2 c_{2}+2 \tilde{k}_{3} \epsilon$. Define $\chi=[e, \widetilde{W}]$, if we pick $\epsilon$ such that

$$
\left\{V_{2} \leq 2 c_{2}+\tilde{k}_{3} \epsilon\right\} \subset\left\{\|\chi\| \leq \frac{\mu}{2}\right\},
$$

then there is a finite time $\widetilde{T}(\mu)$ such that for all $0<\epsilon \leq \epsilon_{4}$

$$
\|\chi\| \leq \frac{\mu}{2}, \quad \forall t \geq \tilde{T}(\mu) .
$$

Since $\eta$ is of order $\epsilon$ in the set $\Lambda$, it follows that there exist an $\epsilon=\epsilon_{5}$ and a finite time $\bar{T}(\mu)$ such that $\|\eta\| \leq \mu / 2 \forall t \geq$ $\bar{T}(\mu)$. Therefore, taking $\epsilon_{2}^{*}=\min \left\{\epsilon_{4}, \epsilon_{5}\right\}$ ensures that there is a finite time $T_{2}=\max \{\tilde{T}, \bar{T}\}$ such that $\|\chi\| \leq \mu / 2$ and $\|\hat{e}\| \leq \mu / 2 \forall t \geq T_{2}$.

Finally, we can establish the exponential converge of the trajectories of the closed-loop system to a small adjustable neighbourhood of the origin. To do this, we consider the Lyapunov function

$$
V=V_{2}+W(\eta)
$$

Its derivative is such that

$$
\dot{V} \leq-k_{1} V_{2}+c_{2}+k_{3}\|\eta\|-\frac{1}{\epsilon}\|\eta\|^{2}+2 \lambda_{\max }\left(P_{0}\right) k_{2}\|\eta\| .
$$

By completing the squares, we get

$$
\begin{aligned}
\dot{V} \leq & -k_{1} V_{2}-\frac{1}{\epsilon}\|\eta\|^{2}+\left(\frac{k_{4}}{2}+\frac{k_{5}}{2}\right)\|\eta\|^{2}+c_{2} \\
& +\frac{k_{3}^{2}}{2 k_{4}}+\frac{2\left(\lambda_{\max }\left(P_{0}\right) k_{2}\right)^{2}}{k_{5}} .
\end{aligned}
$$

Therefore, there exists $0<\epsilon \leq \epsilon_{3}^{*} \leq \epsilon_{2}^{*}$ such that

$$
-\frac{1}{\epsilon}+\frac{k_{4}}{2}+\frac{k_{5}}{2} \leq-k_{6}<0 \text {. }
$$

As a result, we obtain

$$
\dot{V} \leq-k V_{2}-k_{6}\|\eta\|^{2}+c_{2}
$$

or

$$
\dot{V} \leq-k_{m} V+c_{2}
$$

where $k_{m}=\min \left\{k_{1}, k_{6} / \lambda_{\max }\left(P_{0}\right)\right\}$. Using the results above, we guarantee that every trajectory of the system starting in $S \times O$ enters a small neighborhood of the origin in finite time and converges exponentially fast to a small adjustable neighborhood of the origin. This completes the proof.

\section{REFERENCES}

[1] E. Ott, C. Grebogi, and J. A. Yorke, "Controlling chaos," Physical Review Letters, vol. 64, no. 11, pp. 1196-1199, 1990.

[2] A. Garfinkel, M. L. Spano, W. L. Ditto, and J. N. Weiss, "Controlling cardiac chaos," Science, vol. 257, no. 5074, pp. 1230-1235, 1992.

[3] D. J. Christini and J. J. Collins, "Using chaos control and tracking to suppress a pathological nonchaotic rhythm in a cardiac model," Physical Review E, vol. 53, no. 1, pp. R49-R52, 1996.

[4] K. Hall, D. J. Christini, M. Tremblay, J. J. Collins, L. Glass, and J. Billette, "Dynamic control of cardiac alternans," Physical Review Letters, vol. 78, no. 23, pp. 4518-4521, 1997.

[5] D. J. Christini and J. J. Collins, "Real-time, adaptive, modelindependent control of low-dimensional chaotic and nonchaotic dynamical systems," IEEE Transactions on Circuits and Systems I, vol. 44, no. 10, pp. 1027-1030, 1997.

[6] N. Sadegh, "A perceptron network for functional identification and control of nonlinear systems," IEEE Transactions on Neural Networks, vol. 4, no. 6, pp. 982-988, 1993.

[7] K. S. Narendra, "Adaptive control using neural networks," in Neural Networks for Control, W. T. Miller, R. S. Sutton, and P. J. Werbos, Eds., pp. 115-142, MIT Press, Cambridge, Mass, USA, 1991.

[8] K. S. Narendra and K. Parthasarathy, "Identification and control of dynamical systems using neural networks," IEEE Transactions on Neural Networks, vol. 1, no. 1, pp. 4-27, 1990.

[9] F.-C. Chen and H. K. Khalil, "Adaptive control of nonlinear systems using neural networks," International Journal of Control, vol. 55, no. 6, pp. 1299-1317, 1992.

[10] M. M. Polycarpou, "Stable adaptive neural control scheme for nonlinear systems," IEEE Transactions on Automatic Control, vol. 41, no. 3, pp. 447-451, 1996.

[11] S. Seshagiri and H. K. Khalil, "Output feedback control of nonlinear systems using RBF neural networks," IEEE Transactions on Neural Networks, vol. 11, no. 1, pp. 69-79, 2000.

[12] G. A. Rovithakis and M. A. Christodoulou, "Adaptive control of unknown plants using dynamical neural networks," IEEE Transactions on Systems, Man and Cybernetics, vol. 24, no. 3, pp. 400-412, 1994.

[13] F. L. Lewis, S. Jagannathan, and A. Yeşildirek, Neural Network Control of Robot Manipulators and Nonlinear Systems, Taylor and Francis, Philadelphia, Pa, USA, 1999.

[14] F. L. Lewis, A. Yeşildirek, and K. Liu, "Multilayer neural-net robot controller with guaranteed tracking performance," IEEE Transactions on Neural Networks, vol. 7, no. 2, pp. 388-399, 1996. 
[15] S. S. Ge, T. H. Lee, and C. J. Harris, Adaptive Neural Network Control of Robotic Manipulators, World Scientific, Singapore, 1998.

[16] E. B. Kosmatopoulos, M. M. Polycarpou, M. A. Christodoulou, and P. A. Ioannou, "High-order neural network structures for identification of dynamical systems," IEEE Transactions on Neural Networks, vol. 6, no. 2, pp. 422-431, 1995.

[17] K.-I. Funahashi, "On the approximate realization of continuous mappings by neural networks," Neural Networks, vol. 2, no. 3, pp. 183-192, 1989.

[18] A. Teel and L. Praly, "Tools for semiglobal stabilization by partial state and output feedback," SIAM Journal on Control and Optimization, vol. 33, no. 5, pp. 1443-1488, 1995.

[19] H. K. Khalil, Nonlinear Systems, Prentice Hall, Englewood Cliffs, NJ, USA, 2002.

[20] B. D. Anderson, R. R. Bitmead, C. R. J. Johnson, et al., Stability of Adaptive Sysetms: Passivity and Averaging Analysis, MIT Press, Cambridge, Mass, USA, 1986.

[21] A. N. Atassi and H. K. Khalil, "A separation principle for the stabilization of a class of nonlinear systems," IEEE Transactions on Automatic Control, vol. 44, no. 9, pp. 1672-1687, 1999.

[22] H. O. Wang, D. Chen, and L. G. Bushnell, "Control of bifurcations and chaos in heart rhythms," in Proceedings of the 36th IEEE Conference on Decision and Control (CDC'97), vol. 1, pp. 395-400, San Diego, Calif, USA, December 1997.

[23] H. O. Wang, D. Chen, and G. Chen, "Bifurcation control of pathological heart rhythms," in Proceedings of the IEEE International Conference on Control Applications (CCA '98), vol. 2, pp. 858-862, Trieste, Italy, September 1998.

[24] B. van der Pol and J. van der Mark, "The heartbeat considered as a relaxation oscillation and an electrical model of the heart," Philosophical Magazine, vol. 6, pp. 763-775, 1928.

[25] B. J. West, A. L. Goldberger, G. Rovner, and V. Bhargava, "Nonlinear dynamics of the heartbeat. I. The AV junction: passive conduit or active oscillator?" Physica D, vol. 17, no. 2, pp. 198-206, 1985.

[26] W. L. Keith and R. H. Rand, "1:1 and 2:1 phase entrainment in a system of two coupled limit cycle oscillators," Journal of Mathematical Biology, vol. 20, no. 2, pp. 133-152, 1984.

[27] D. di Bernardo, M. G. Signorini, and S. Cerutti, "A model of two nonlinear coupled oscillators for the study of heartbeat dynamics," International Journal of Bifurcation and Chaos, vol. 8, no. 10, pp. 1975-1985, 1998.

[28] J.-B. Pomet and L. Praly, "Adaptive nonlinear regulation: estimation from the Lyapunov equation," IEEE Transactions on Automatic Control, vol. 37, no. 6, pp. 729-740, 1992. 

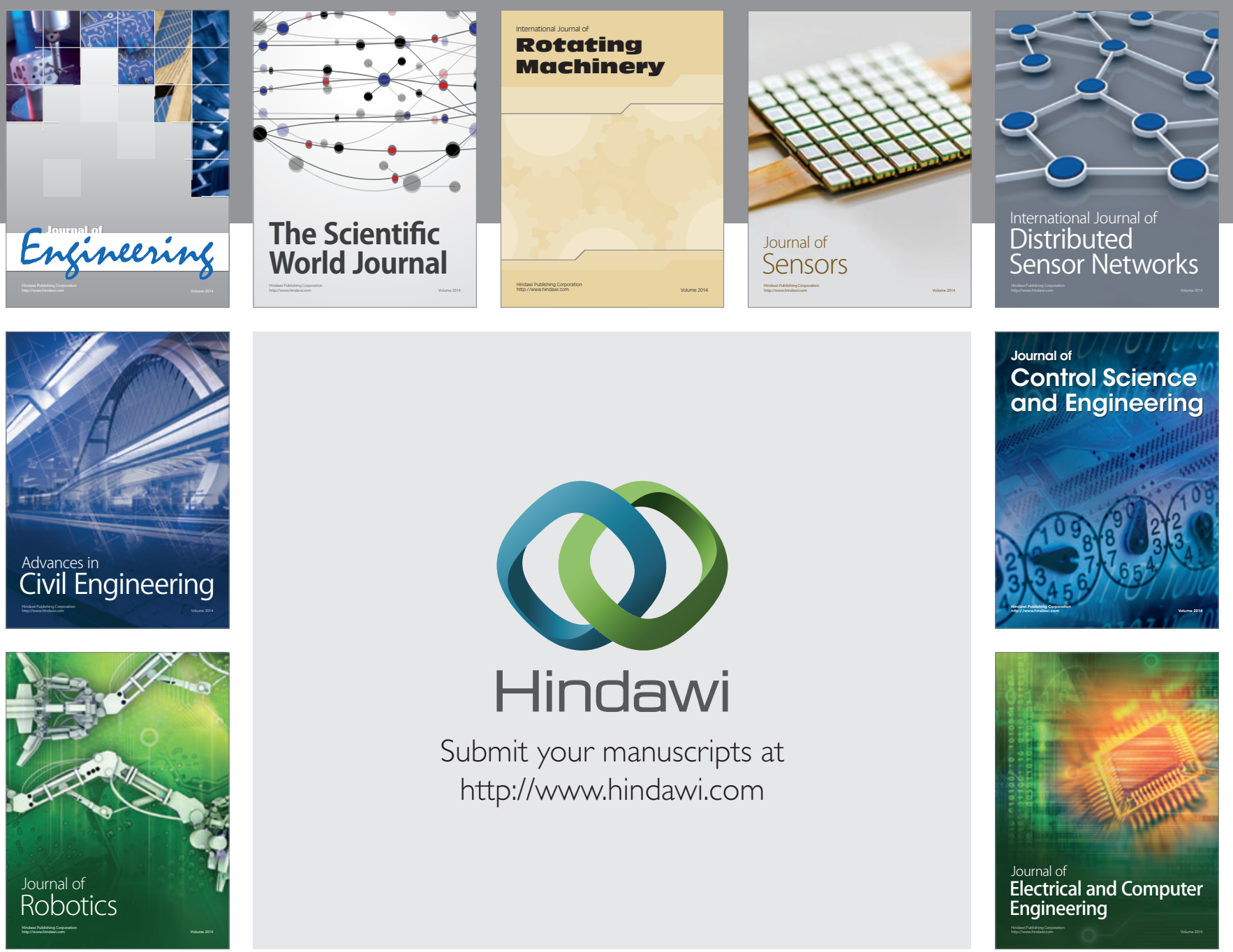

Submit your manuscripts at

http://www.hindawi.com
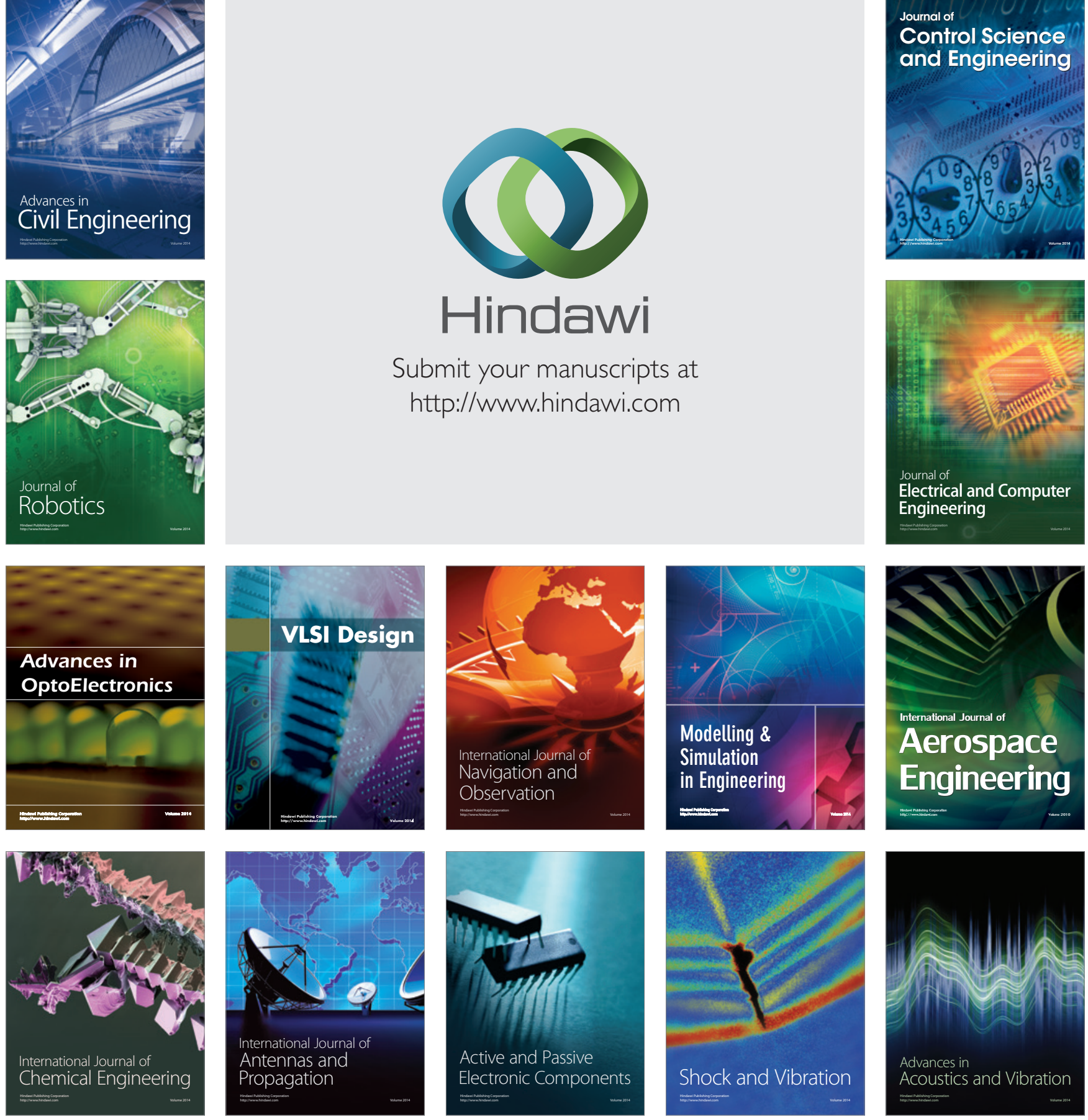